\title{
Total Synthesis of 7-Deoxypancratistatin-1- Carboxaldehyde and Carboxylic Acid via Solvent-free Intramolecular Aziridine Opening: Phenanthrene to Phenanthridone Cyclization Strategy.
}

Jonathan Collins, Melissa Drouin, Xuetong Sun, Uwe Rinner, and Tomas Hudlicky ${ }^{*}$

Department of Chemistry and Centre for Biotechnology, Brock University, 500 Glenridge Ave., St. Catharines, ON, L2S 3A1, Canada thudlicky@brocku.ca

\section{Contents of Supporting information}

Contents

General Experimental Section

Characterizations

Compound 12

Compound $\mathbf{6}$

Compound 7

Compound 13

Compound 16

Compound 17

Compound 18

Compound 19

Compound 20

Spectral data
Page Number

2

2

2

3

4

5

6

7

8

10

10

11 


\section{General Experimental section}

All non-aqueous reactions were conducted in an argon atmosphere using standard schlenk techniques for the exclusion of moisture and air. Methylene chloride was distilled from calcium hydride, THF and toluene were dried over potassium/benzophenone. Analytical thin layer chromatography was performed on Silicycle $60 \AA 250 \mu \mathrm{m}$ TLC plates with F-254 indicator. Flash column chromatography was performed using Kieselgel 60 (230-400 mesh). Melting points were recorded on a Hoover Unimelt apparatus and are uncorrected. IR spectra were obtained on a Perkin-Elmer One FT-IR spectrometer. Optical rotation was measured on a Perkin-Elmer 341 polarimeter at a wavelength of $589 \mathrm{~nm} .{ }^{1} \mathrm{H}$ and ${ }^{13} \mathrm{C}$ spectra were recorded on a $300 \mathrm{MHz}$ and $600 \mathrm{MHz}$ spectrometer. All chemical shift are referenced to TMS or residual undeuterated solvent. Data of proton spectra are reported as follows: chemical shift (multiplicity [singlet (s), doublet (d), triplet (t), quartet (q) and multiplet (m)], coupling constants [Hz], integration). Carbon spectra were recorded with complete proton decoupling and the chemical shifts are reported in ppm $(\delta)$ relative to solvent resonance as internal standard. Mass spectra and high resolution mass spectra were performed by the analytical division at Brock University.

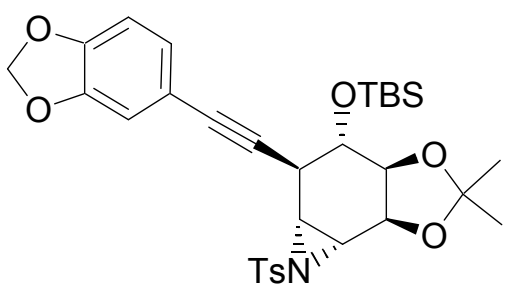

(1S,2R,3R,4R,5S,6R)-3,4-(Isopropylidenedioxy)-5-[(tert-butyldimethylsilyl)oxy]-6-2Benzo[1.3]dioxol-5-ylethynyl-(4'-methylphenylsulfonyl)-7-azabicyclo[4.1.0]heptane (12). To a solution of acetylene $11(2.74 \mathrm{~g}, 18.75 \mathrm{mmol})$ in $18 \mathrm{~mL}$ dry toluene was added at $-78{ }^{\circ} \mathrm{C} 8.33 \mathrm{~mL}$ of a solution of $\mathrm{nBuLi}$ in hexanes $(2.25 \mathrm{M}, 18.75 \mathrm{mmol})$. The solution was stirred for 10 minutes before $18.75 \mathrm{~mL}$ of a solution of $\mathrm{Me}_{2} \mathrm{AlCl}\left(1.0 \mathrm{M}\right.$ in $\mathrm{CH}_{2} \mathrm{Cl}_{2}$, $18.75 \mathrm{mmol}$ ) was added dropwise. The reaction flask was allowed to warm to room temperature and stir for $1 \mathrm{~h}$. The reaction flask was then cooled to $-20^{\circ} \mathrm{C}$ and $18 \mathrm{~mL}$ of a solution of epoxide $5(3.16 \mathrm{~g}, 9.38 \mathrm{mmol})$ in toluene was added dropwise over $20 \mathrm{~min}$. The reaction was stirred at $-20^{\circ} \mathrm{C}$ for $3.5 \mathrm{~h}$ before being place in an ice bath and allowed to slowly warm to room temperature and stir for $12 \mathrm{~h}$. The reaction was cooled in an ice bath and quenched with $1 \mathrm{M} \mathrm{HCl}$. Ethyl acetate $(200 \mathrm{~mL})$ was added and the layers where separated. The aqueous phase was extracted $3 \times 100 \mathrm{~mL}$ EtOAc and the combined organic layers dried over $\mathrm{Na}_{2} \mathrm{SO}_{4}$. Concentration under reduced pressure and purification by flash column chromatography (hexanes:ethyl acetate, 7:1 to 4:1) afforded alcohol intermediate which was immediately subjected to protection protocol $(2.01 \mathrm{~g}, 44 \%)$; $[\alpha]^{22}{ }_{\mathrm{D}}-113.05\left(c 0.5, \mathrm{CHCl}_{3}\right) ; \mathrm{R}_{\mathrm{f}} 0.30$ (hexanes:ethyl acetate 2:1); IR (film) $v$ 3491, 2988, $1163 \mathrm{~cm}^{-1} ;{ }^{1} \mathrm{H}$ NMR $\left(300 \mathrm{MHz}, \mathrm{CDCl}_{3}\right) \delta: 7.78(\mathrm{~d}, J=8.1 \mathrm{~Hz}, 2 \mathrm{H}), 7.38(\mathrm{~d}, J=8.1 \mathrm{~Hz}$, 
2H), $6.91(\mathrm{dd}, J=8.2 \mathrm{~Hz}, 1.8 \mathrm{~Hz} 1 \mathrm{H}), 6.83(\mathrm{~d}, J=1.5 \mathrm{~Hz}, 1 \mathrm{H}), 6.73(\mathrm{~d}, J=7.9 \mathrm{~Hz}, 1 \mathrm{H})$, $5.97(\mathrm{~s}, 2 \mathrm{H}), 4.47(\mathrm{~d}, J=6.4 \mathrm{~Hz}, 1 \mathrm{H}), 4.22(\mathrm{dd}, J=6.1,4.4 \mathrm{~Hz}, 1 \mathrm{H}), 3.98(\mathrm{~m}, 1 \mathrm{H}), 3.40$ $(\mathrm{d}, J=6.4 \mathrm{~Hz}, 1 \mathrm{H}), 3.24(\mathrm{~m}, 2 \mathrm{H}), 3.06(\mathrm{~d}, J=9.6 \mathrm{~Hz}, 1 \mathrm{H}), 2.47(\mathrm{~s}, 3 \mathrm{H}), 1.49(\mathrm{~s}, 3 \mathrm{H})$, 1.32 (s, $3 \mathrm{H}) \mathrm{ppm} ;{ }^{13} \mathrm{C}$ NMR $\left(75 \mathrm{MHz}, \mathrm{CDCl}_{3}\right) \delta: 148.1,147.5,145.7,134.2,130.4$, $128.1,126.4,116.2,111.8,110.3,108.6,101.5,84.2,83.8,75.4,70.1,68.7,42.3,40.5$, 31.1, 27.4, 25.2, 21.9 ppm; HRMS $\left(F A B M^{+}\right.$) calcd for $\mathrm{C}_{25} \mathrm{H}_{25} \mathrm{NO}_{7} \mathrm{~S} 484.1430$, found 484.1428 .

Alcohol intermediate (240mg, $0.49 \mathrm{mmol}$ ) was dissolved in $5 \mathrm{~mL}$ of $\mathrm{CH}_{2} \mathrm{Cl}_{2}$ and triethylamine $(0.14 \mathrm{~mL}, 1.04 \mathrm{mmol})$ was added. The reaction flask was cooled to $-78{ }^{\circ} \mathrm{C}$ and $t$-butyldimethylsilytriflate $(0.12 \mathrm{~mL}, 0.546 \mathrm{mmol})$ was added dropwise to the stirring solution. After stirring for 30 minutes at $-78{ }^{\circ} \mathrm{C}$ the reaction was quenched with water and the two phases separated. The aqueous phase was extracted with $\mathrm{CH}_{2} \mathrm{Cl}_{2}(2 \times 15$ $\mathrm{mL}$ ) and the combined organic solution was washed sequentially with $5 \%$ citric acid (2 $\mathrm{mL})$ and brine $(2 \mathrm{~mL})$ before drying over sodium sulfate. The solvent was removed under reduced pressure and the residue was purified by flash column chromatography (hexane:ethyl acetate, 9:1 to $2: 1)$ affording $12(0.276 \mathrm{~g}, 93 \%)$ as a colorless oil.; $[\alpha]^{24}{ }_{\mathrm{D}}$ +57.7 (c 0.5, $\mathrm{CHCl}_{3}$ ); $\mathrm{R}_{\mathrm{f}} 0.49$ (hexanes:ethyl acetate, 2:1); IR (film) v 2953, 2929, 2892, $2856,1599,1490 \mathrm{~cm}^{-1} ;{ }^{1} \mathrm{H}$ NMR $\left(300 \mathrm{MHz}, \mathrm{CDCl}_{3}\right) \delta: 7.83(\mathrm{~d}, J=8.1 \mathrm{~Hz}, 2 \mathrm{H}), 7.38(\mathrm{~d}, J$ $=8.1 \mathrm{~Hz}, 2 \mathrm{H}), 6.94(\mathrm{~d}, J=8.1 \mathrm{~Hz}, 1 \mathrm{H}), 6.84(\mathrm{~s}, 1 \mathrm{H}), 6.77(\mathrm{~d}, J=8.1 \mathrm{~Hz}, 1 \mathrm{H}), 5.99(\mathrm{~s}$, $2 \mathrm{H}), 4.45(\mathrm{~d}, J=5.1 \mathrm{~Hz}, 1 \mathrm{H}), 3.83(\mathrm{~m}, 2 \mathrm{H}), 3.26(\mathrm{~m}, 2 \mathrm{H}), 2.84(\mathrm{~d}, J=7.5 \mathrm{~Hz}), 2.47$ (s, $3 \mathrm{H}), 1.52(\mathrm{~s}, 3 \mathrm{H}), 1.35(\mathrm{~s}, 3 \mathrm{H}), 0.87(\mathrm{~s}, 9 \mathrm{H}), 0.11(\mathrm{~s}, 6 \mathrm{H}) \mathrm{ppm} ;{ }^{13} \mathrm{C}$ NMR $(75 \mathrm{MHz}$, $\left.\mathrm{CDCl}_{3}\right) \delta$ : 147.8, 147.3, 134.7, 129.8, 127.9, 126.1, 111.6, 109.7, 108.4, 101.3, 86.3, 83.5, 71.7, 43.2, 39.53, 34.58, 27.9, 25.8, 25.79, 25.7, 21.7, 18.12, -4.4, -4.7 ppm; HRMS-EI Calcd for $\mathrm{C}_{30} \mathrm{H}_{36} \mathrm{NO}_{7} \mathrm{SSi}$ : 540.1481; Found, 540.1487; Anal. calcd for $\mathrm{C}_{31} \mathrm{H}_{39} \mathrm{NO}_{7} \mathrm{SSi} \mathrm{C}$, $62.28 ; \mathrm{H}, 6.58$; found $\mathrm{C}, 62.22 ; \mathrm{H}, 6.73$

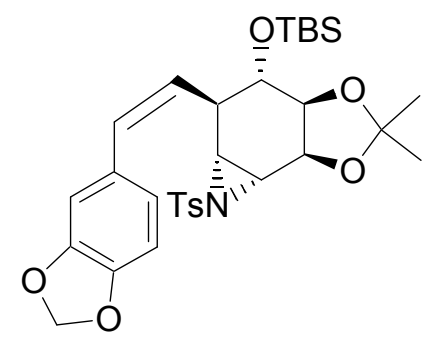

(1S,2R,3R,4R,5S,6R)-3,4-(Isopropylidenedioxy)-5-[(tert-butyldimethylsilyl)oxy]-6-2Benzo[1,3]dioxol-5-ylethenyl-(4'-methylphenylsulfonyl)-7-azabicyclo[4.1.0]heptane

(6). To a $1.0 \mathrm{M}$ solution of $\mathrm{BH}_{3}$.THF complex $(2.5 \mathrm{~mL}, 2.5 \mathrm{mmol})$ was added cyclohexene $(0.484 \mathrm{~mL}, 4.77 \mathrm{mmol})$ at $0{ }^{\circ} \mathrm{C}$. After 10 minutes a heavy precipitate was formed. The reaction mixture was kept at $0{ }^{\circ} \mathrm{C}$ for $1 \mathrm{~h}$ before acetylene derivative $\mathbf{1 2}$ $(0.356 \mathrm{mg}, 0.596 \mathrm{mmol})$ in $4.5 \mathrm{~mL}$ of THF was added. The reaction mixture was stirred at $0{ }^{\circ} \mathrm{C}$ until total consumption of starting material ( $2 \mathrm{~h}$, TLC) before being quenched with $1 \mathrm{~mL}$ HOAc. $60 \mathrm{~mL}$ EtOAc were added and the reaction mixture was washed with saturated aq. $\mathrm{NaHCO}_{3}(2 \times 15 \mathrm{~mL}), \mathrm{H}_{2} \mathrm{O}(2 \times 15 \mathrm{~mL})$, and brine $(10 \mathrm{~mL})$ before drying over $\mathrm{Na}_{2} \mathrm{SO}_{4}$. The solvent was removed under reduced pressure and the residue was 
purified by flash column chromatography (hexanes:ethyl acetate, 8:1) affording $0.271 \mathrm{~g}$ of $6(76 \%)$; $[\alpha]^{23}{ }_{\mathrm{D}}-26.14\left(\mathrm{c} 1.0, \mathrm{CHCl}_{3} ; R_{\mathrm{f}} 0.35\right.$ (hexanes:ethyl acetate, $\left.4: 1\right)$; IR (film) $v$ 2986, 2930, 2894, 2856, 1598,1489 $\mathrm{cm}^{-1} ;{ }^{1} \mathrm{H}$ NMR (300 MHz, $\left.\mathrm{CDCl}_{3}\right) \delta: 7.78$ (d, $J=8.1$ $\mathrm{Hz}, 2 \mathrm{H}), 7.29(\mathrm{~d}, J=8.1 \mathrm{~Hz}, 2 \mathrm{H}), 6.65(\mathrm{~m}, 3 \mathrm{H}), 6.51(\mathrm{~d}, J=11.7 \mathrm{~Hz}, 1 \mathrm{H}), 5.97(\mathrm{~s}, 2 \mathrm{H})$, $5.54(\mathrm{t}, J=11.3 \mathrm{~Hz}, 1 \mathrm{H}), 4.43(\mathrm{~d}, J=6,1 \mathrm{H}), 3.85(\mathrm{t}, J=6.3,1 \mathrm{H}), 3.61(\mathrm{t}, J=7.2 \mathrm{~Hz})$, $3.18(\mathrm{~d}, J=6.6,1 \mathrm{H}), 2.91(\mathrm{~m}, 2 \mathrm{H}), 2.44(\mathrm{~s}, 3 \mathrm{H}), 1.52(\mathrm{~s}, 3 \mathrm{H}), 1.33(\mathrm{~s}, 3 \mathrm{H}), 0.79(\mathrm{~s}, 9 \mathrm{H})$, $0.02(\mathrm{~s}, 3 \mathrm{H}),-0.04(\mathrm{~s}, 3 \mathrm{H}) \mathrm{ppm} ;{ }^{13} \mathrm{C} \mathrm{NMR}\left(150 \mathrm{MHz}, \mathrm{CDCl}_{3}\right) \delta: 147.5,146.6,144.6$, 134.7, 132.0, 130.3, 129.8, 129.7, 129.5, 128.5, 127.9, 122.5, 109.35, 109.0, 108.1, 100.9, 83.2, 78.0, 72.6, 71.8, 43.7, 39.9, 39.5, 30.1, 27.8, 25.8, 25.79. 25.75, 25.72, 25.51, 23.7, 21.7, 18.1, -4.3, -4.7 ppm; HRMS-EI Calcd for $\mathrm{C}_{31} \mathrm{H}_{41} \mathrm{NO}_{7} \mathrm{SSi}$ : 599.2373; Found, 599.2376; Anal. calcd for $\mathrm{C}_{31} \mathrm{H}_{41} \mathrm{NO}_{7} \mathrm{SSi}$ C, 62.28; H, 6.58; found C, 61.30; H, 6.63

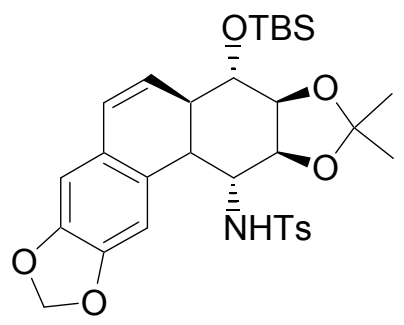

\section{$N$-[(1R,2aS,4aS,5S,5aR,12bR)-5-(tert-Butyl-dimethyl-silanyloxy)-3,3-dimethyl- 1,2a,4a,5,5a,12b-hexahydro-phenanthro[2,3-d][1,3] dioxol-1-yl]4-methyl-}

benzenesulfonamide (7). A flame-dried 25-mL flask was charged with olefin 13 (336 $\mathrm{mg}, 0.561 \mathrm{mmol})$ and silica gel which has been activate by heating under vacuum at 120 ${ }^{\circ} \mathrm{C}$ overnight $(1.5 \mathrm{~g})$. The starting materials were suspended in $10 \mathrm{~mL}$ freshly distilled methylene chloride and the solvent removed under reduced pressure. The silica gel supporting the absorbed reactants was heated externally at $120{ }^{\circ} \mathrm{C}$ under nitrogen atmosphere for $24 \mathrm{~h}$, after which time the silica gel was loaded onto flash silica gel column and eluted with hexanes:ethyl acetate, $8: 1-5: 1$ to give $175 \mathrm{mg}(52 \%)$ of olefin 7 as a clear and colorless oil.; $[\alpha]^{23}-123.7\left(c 1.0, \mathrm{CHCl}_{3}\right) ; R_{\mathrm{f}} 0.35$ (hexanes: ethyl acetate, 2:1); IR (film) v 3268, 2929, 2887, 2857, 1598,1503, $1485 \mathrm{~cm}^{-1} ;{ }^{1} \mathrm{H}$ NMR $(600 \mathrm{MHz}$, $\left.\mathrm{CDCl}_{3}\right) \delta: 7.43(\mathrm{~d}, J=7 \mathrm{~Hz}, 2 \mathrm{H}), 7.13(\mathrm{~d}, J=7 \mathrm{~Hz}, 2 \mathrm{H}), 6.49(\mathrm{~s}, 2 \mathrm{H}), 6.34(\mathrm{~d}, J=8 \mathrm{~Hz}$, $1 \mathrm{H}), 5.95(\mathrm{~s}, 1 \mathrm{H}), 5.86(\mathrm{~s}, 1 \mathrm{H}), 5.76(\mathrm{~d}, J=8 \mathrm{~Hz}, 1 \mathrm{H}), 4.51(\mathrm{~d}, J=7 \mathrm{~Hz}, 1 \mathrm{H}), 4.28(\mathrm{~m}$, $1 \mathrm{H}), 4.11(\mathrm{~m}, 1 \mathrm{H}), 3.99(\mathrm{~m}, 1 \mathrm{H}), 3.79(\mathrm{~m}, 1 \mathrm{H}), 2.82(\mathrm{~m}, 1 \mathrm{H}), 2.62(\mathrm{dd}, J=11.1 \mathrm{~Hz}, J=$ $5.4 \mathrm{~Hz}, 1 \mathrm{H}), 2.40(\mathrm{~s}, 3 \mathrm{H}), 1.43(\mathrm{~s}, 3 \mathrm{H}), 1.33(\mathrm{~s}, 3 \mathrm{H}), 0.89(\mathrm{~s}, 9 \mathrm{H}), 0.11(\mathrm{~s}, 3 \mathrm{H}), 0.07$ (s, $3 \mathrm{H}) \mathrm{ppm} ;{ }^{13} \mathrm{C} \mathrm{NMR}\left(150 \mathrm{MHz}, \mathrm{CDCl}_{3}\right) \delta: 146.7,145.9,142.1,138.9,128.9,128.6$, 127.7, 126.8, 126.3, 126.2, 110.4, 109.2, 107.0, 79.0, 78.3, 70.3, 54.1, 42.5, 41.5, 38.9, 27.8, 26.3, 25.7, 25.3, 22.7, 21.5, 18.0, -5.0, -5.0 ppm; HRMS-EI Calcd for $\mathrm{C}_{31} \mathrm{H}_{41} \mathrm{NO}_{7} \mathrm{SSi}$ : 599.2373; Found, 599.2370; Anal. calcd for $\mathrm{C}_{31} \mathrm{H}_{41} \mathrm{NO}_{7} \mathrm{SSi} \mathrm{C}, 62.07$; $\mathrm{H}$, 6.89; found $\mathrm{C}, 62.16$; $\mathrm{H}, 6.94$ 


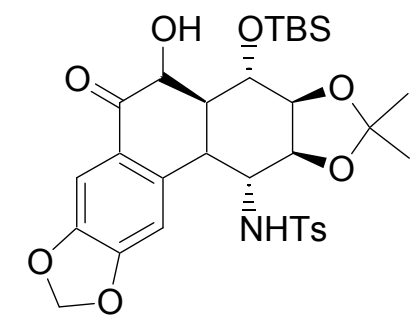

\section{$N$-[(1R,2aS,4aS,5S,5aS,12bR)-5-(tert-Butyl-dimethyl-silanyloxy)-6-hydroxy-3,3-} dimethyl-7-oxo-1,2a,4a,5,5a,6,7,12b-octahydro-phenanthro[2,3- $d][1,3]$ dioxol-1-yl]4methyl-benzenesulfonamide (13). To a solution of olefin $7(0.240 \mathrm{mg}, 0.4 \mathrm{mmol})$ in methylene chloride $(10 \mathrm{~mL})$ was added 4-methylmorpholine $N$-oxide $(58 \mathrm{mg}, 0.48$ mmol). The reaction mixture was allowed to stir for 10 minutes before the introduction of a single crystal of osmium tetroxide and two drops of water. The reaction was stirred until total consumption of starting material $(10 \mathrm{~h})$ before being quenched with a saturated solution of saturated sodium bisulfite $(6 \mathrm{~mL})$. The two layers were separated and the aqueous phase was extracted with ethyl acetate $(3 \times 30 \mathrm{~mL})$. The organic phase was dried over sodium sulfate, filtered, and concentrated in vacuo to provide hydroxyketone 13 as a white crystalline solid $(0.227 \mathrm{~g}, 89 \%)$ that was used without further purification; $R_{\mathrm{f}} 0.42$ (hexanes:ethyl acetate, $1: 1$ ); $\mathrm{mp}>200{ }^{\circ} \mathrm{C}$; IR (film) $v 3478,3263,2929,2857$, $1670,1614,1504,1482,1444,1386,1330,1252,1218,1156,1075,1039 \mathrm{~cm}^{-1} ;{ }^{1} \mathrm{H}$ NMR $\left(600 \mathrm{MHz}, \mathrm{CDCl}_{3}\right) \delta: 7.54(\mathrm{~d}, J=7.8 \mathrm{~Hz}, 2 \mathrm{H}), 7.49,(\mathrm{~s}, 1 \mathrm{H}), 7.18(\mathrm{~d}, J=7.8 \mathrm{~Hz}, 2 \mathrm{H})$, $6.70(\mathrm{~s}, 1 \mathrm{H}), 6.07(\mathrm{~s}, 1 \mathrm{H}), 6.00(\mathrm{~s}, 1 \mathrm{H}), 4.79(\mathrm{~d}, J=8.7 \mathrm{~Hz}, 1 \mathrm{H}), 4.71(\mathrm{~m}, 2 \mathrm{H}), 4.19(\mathrm{~m}$, $1 \mathrm{H}), 4.08(\mathrm{~m}, 1 \mathrm{H}), 3.74(\mathrm{~m}, 2 \mathrm{H}), 3.08(\mathrm{dd}, J=10.2 \mathrm{~Hz}, J=1.8 \mathrm{~Hz}, 1 \mathrm{H}), 2.45(\mathrm{~m}, 1 \mathrm{H})$, $2.41(\mathrm{~s}, 3 \mathrm{H}), 1.36(\mathrm{~s}, 3 \mathrm{H}), 1.31(\mathrm{~s}, 3 \mathrm{H}), 0.87(\mathrm{~s}, 9 \mathrm{H}), 0.12(\mathrm{~s}, 3 \mathrm{H}), 0.07(\mathrm{~s}, 3 \mathrm{H}) \mathrm{ppm} ;{ }^{13} \mathrm{C}$ NMR (150 MHz, $\left.\mathrm{CDCl}_{3}\right) \delta: 196.6,152.5,147.9,142.6,140.5,138.9,129.1,126.9,124.7$, $111.2,109.6,106.9,102.1,78.9,78.7,70.3,65.9,57.9,49.4,39.7,27.9,25.7,21.5,17.95$, $-5.1 \mathrm{ppm}$; HRMS-EI Calcd for $\mathrm{C}_{27} \mathrm{H}_{32} \mathrm{NO}_{9} \mathrm{SSi}\left(\mathrm{M}^{+}-57\right): 574.1567$, Found: 574.1572 


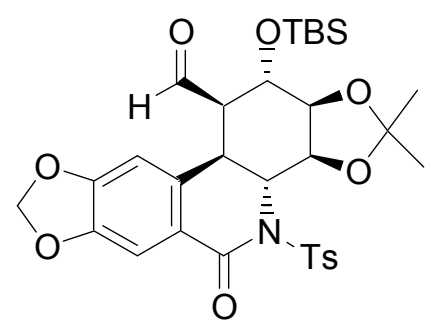

(3aS,3bR,10bR,11R,12S,12aS)-12-(tert-Butyl-dimethyl-silanyloxy)-2,2-dimethyl-5oxo-4-(toluene-4-sulfonyl)-3a,3b,4,5,10b,11,12,12a-octahydro-1,3,7,9-tetraoxa-4-azadicyclopenta $[\boldsymbol{a}, \boldsymbol{h}]$ phenanthrene-11-carbaldehyde (16). To a $10 \mathrm{~mL}$ round bottomed flask was added hydroxyl ketone $\mathbf{1 3}(0.4 \mathrm{~g}, 0.63 \mathrm{mmol})$ and $6 \mathrm{~mL}$ of a 1:1 mixture of ethanol:dioxane. The reaction flask was cooled externally in an ice bath and $\mathrm{NaBH}_{4}(24$ $\mathrm{mg}, 0.63 \mathrm{mmol}$ ) was added in one portion. The reaction was removed from the bath and allowed to warm to room temperature over $1 \mathrm{~h}$. The reaction was quenched with $1 \mathrm{~N} \mathrm{HCl}$ $(4 \mathrm{~mL})$ and separated. The aqueous phase was extracted with $\mathrm{CH}_{2} \mathrm{Cl}_{2}(3 \times 20 \mathrm{~mL})$ and the organic phase combined before drying over sodium sulfate. The crude mixture was concentrated in a $25 \mathrm{~mL}$ round bottomed flash and taken up in dioxane $(8 \mathrm{~mL})$. A stirring bar was added and the reaction was stirred while sodium periodate $(0.332,1.5 \mathrm{mmol})$ was added. The flask was covered to exclude light and $\mathrm{H}_{2} \mathrm{O}$ ( 15 drops) added. The reaction was stirred until total consumption of starting material (23h) as monitored by TLC. The reaction was quenched with $\mathrm{H}_{2} \mathrm{O}(10 \mathrm{~mL})$ and separated. The aqueous phase was extracted with $\mathrm{CH}_{2} \mathrm{Cl}_{2}(3 \times 50 \mathrm{~mL})$ and the combined organic phases dried over sodium sulfate. Concentration provided 17.

To a solution of hemi-aminal 17 (394 mg, $0.62 \mathrm{mmol}$ ) in $N, N$-Dimethylformamide (3 $\mathrm{mL}$ ) was added 2-Iodoxybenzoic acid $(520 \mathrm{mg}, 1.86 \mathrm{mmol})$. After total consumption of starting material (by TLC), the reaction mixture was diluted with diethyl ether $(200 \mathrm{~mL})$ and washed sequentially with saturated aqueous sodium bisulfite $(10 \mathrm{~mL})$, sodium bicarbonate $(3 \times 10 \mathrm{~mL}), \mathrm{H}_{2} \mathrm{O}(10 \times 1 \mathrm{~mL})$, and brine $(10 \mathrm{~mL})$. The organic phase was dried over magnesium sulfate, filtered and concentrated. The final product was isolated by column chromatography (hexanes:ethyl acetate, 4:1). Yield: $225 \mathrm{mg}, 61 \%$, white solid; $R_{\mathrm{f}} 0.31$ (hexanes:ethyl acetate, $4: 1$ ); $\mathrm{mp}>200{ }^{\circ} \mathrm{C}$, recrystallized from hexanes/ethyl acetate $4: 1 ;[\alpha]_{\mathrm{D}}{ }^{21}+31.67\left(c 0.5, \mathrm{CHCl}_{3}\right) ;$ IR (film) v 2929, 2857, 1725, 1689, 1619, $1505,1484,1386,1361,1287,1255,1220,1172,1077,1036 \mathrm{~cm}^{-1} ;{ }^{1} \mathrm{H}$ NMR $(600 \mathrm{MHz}$, $\left.\mathrm{CDCl}_{3}\right) \delta: 9.49(\mathrm{~s}, 1 \mathrm{H}), 8.3(\mathrm{~d}, J=8.2 \mathrm{~Hz}, 2 \mathrm{H}), 7.58(\mathrm{~s}, 1 \mathrm{H}), 7.33(\mathrm{~d}, J=8.2 \mathrm{~Hz}, 2 \mathrm{H})$, $7.28(\mathrm{~s}, 1 \mathrm{H}), 6.55(\mathrm{~s}, 1 \mathrm{H}), 6.04(\mathrm{~d}, J=5 \mathrm{~Hz}, 2 \mathrm{H}), 5.81(\mathrm{dd}, J=8.4 \mathrm{~Hz}, J=5.2 \mathrm{~Hz}, 1 \mathrm{H})$, $4.79(\mathrm{~m}, 1 \mathrm{H}), 4.50(\mathrm{dd}, J=12.7 \mathrm{~Hz}, J=8.4 \mathrm{~Hz}, 1 \mathrm{H}), 4.27(\mathrm{dd}, J=5.2 \mathrm{~Hz}, J=2.7 \mathrm{~Hz}$, $1 \mathrm{H}), 3.83(\mathrm{dd}, J=12.6, J=4.0 \mathrm{~Hz}, 1 \mathrm{H}), 3.31(\mathrm{~m}, 1 \mathrm{H}), 2.45(\mathrm{~s}, 3 \mathrm{H}), 1.42(\mathrm{~s}, 3 \mathrm{H}), 1.32(\mathrm{~s}$, $1 \mathrm{H}), 0.99(\mathrm{~s}, 9 \mathrm{H}), 0.26(\mathrm{~s}, 3 \mathrm{H}), 0.25(\mathrm{~s}, 3 \mathrm{H}) \mathrm{ppm} ;{ }^{13} \mathrm{C} \mathrm{NMR}\left(150 \mathrm{MHz}, \mathrm{CDCl}_{3}\right) \delta: 196.2$, 166.0, 153.0, 147.1, 143.9, 138.8, 137.0, 128.9, 128.8, 110.1, 109.4, 104.2, 102.2, 72.4, $66.6,65.5,55.6,35.4,31.0,27.9,26.9,25.7,22.7,21.7,18.1,14.2,-4.7,-4.9 \mathrm{ppm}$; HRMS-EI Calcd for $\mathrm{C}_{30} \mathrm{H}_{36} \mathrm{NO}_{9} \mathrm{SSi}\left(\mathrm{M}^{+}-15\right)$ : 614.1879, Found: 614.1870; Anal. calcd for $\mathrm{C}_{31} \mathrm{H}_{39} \mathrm{NO}_{9} \mathrm{SSi}$ C, 59.12; H, 6.24; found C, 59.31; H, 6.29. 


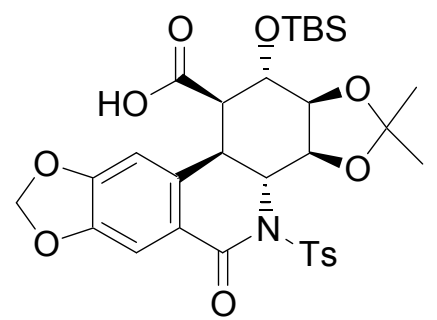

(3aS,3bR,10bR,11R,12S,12aS)-12-(tert-Butyl-dimethyl-silanyloxy)-2,2-dimethyl-5oxo-4-(toluene-4-sulfonyl)-3a,3b,4,5,10b,11,12,12a-octahydro-1,3,7,9-tetraoxa-4-azadicyclopenta $[a, h]$ phenanthrene-11-carboxylic acid (17). To a solution of aldehyde 16 $(144 \mathrm{mg}, 0.229 \mathrm{mmol})$ in dry methylene chloride $(5 \mathrm{~mL})$ was added sodium phosphate dibasic $(81 \mathrm{mg}, 0.57 \mathrm{mmol})$. The suspension was stirred while 3-chloroperbenzoic acid $(130 \mathrm{mg}, 0.57 \mathrm{mmol})$ was added. The reaction flask was sealed and heated at $40{ }^{\circ} \mathrm{C}$ overnight. The reaction mixture was diluted with methylene chloride $(80 \mathrm{~mL})$ and washed sequentially with saturated aqueous sodium bisulfite $(10 \mathrm{~mL})$, sodium bicarbonate $(10 \mathrm{~mL})$, and dried over sodium sulfate. The organic phase was filtered and concentrated in vacuo to provide carboxylic acid $\mathbf{1 7}$ as a white crystalline solid $(0.125 \mathrm{~g}$, $85 \%$ ) that was used without further purification; ; $R_{\mathrm{f}} 0.1$ (hexanes/ethyl acetate, $1: 1$ ); $\mathrm{mp}$ $>200{ }^{\circ} \mathrm{C} ;[\alpha]_{\mathrm{D}}{ }^{22}-35.09\left(c 1.25, \mathrm{CHCl}_{3}\right)$; IR $(\mathrm{KBr})$ v 3246, 2930, 2891, 2857, 1710, 1688, $1619,1505,1484,1361,1240,1220,1172,1078,1033 \mathrm{~cm}^{-1} ;{ }^{1} \mathrm{H}$ NMR $(300 \mathrm{MHz}$, $\left.\mathrm{CDCl}_{3}\right) \delta: 8.29(\mathrm{~d}, J=8.3 \mathrm{~Hz}, 2 \mathrm{H}), 7.53(\mathrm{~s}, 1 \mathrm{H}), 7.32(\mathrm{~d}, J=8.3 \mathrm{~Hz}, 2 \mathrm{H}), 7.28(\mathrm{~s}, 1 \mathrm{H})$, $6.56(\mathrm{~s}, 1 \mathrm{H}), 6.02(\mathrm{~d}, J=3 \mathrm{~Hz}, 2 \mathrm{H}), 5.77(\mathrm{dd}, J=8.30 \mathrm{~Hz}, J=5.3 \mathrm{~Hz}, 1 \mathrm{H}), 4.85(\mathrm{dd}, J=$ $12.5 \mathrm{~Hz}, J=8.4 \mathrm{~Hz}, 1 \mathrm{H})), 4.84(\mathrm{t}, J=4.7 \mathrm{~Hz}, 1 \mathrm{H}), 4.22(\mathrm{dd} J=5.22, J=2.8 \mathrm{~Hz}, 1 \mathrm{H})$, $3.76(\mathrm{dd}, J=12.4 \mathrm{~Hz}, J=4.1 \mathrm{~Hz}, 1 \mathrm{H}), 3.38(\mathrm{t}, J=3.5 \mathrm{~Hz}, 1 \mathrm{H}), 2.45(\mathrm{~s}, 3 \mathrm{H}), 1.40(\mathrm{~s}, 3 \mathrm{H})$, $1.27(\mathrm{~s}, 1 \mathrm{H}), 0.96(\mathrm{~s}, 9 \mathrm{H}), 0.21(\mathrm{~s}, 6 \mathrm{H}) \mathrm{ppm} ;{ }^{13} \mathrm{C} \mathrm{NMR}\left(150 \mathrm{MHz}, \mathrm{CDCl}_{3}\right) \delta: 174.3$, 166.2, 152.8, 146.9, 143.8, 138.9, 137.7, 129.0, 128.8, 122.4, 109.8, 109.2, 103.4, 102.1, 72.8, 68.2, 64.9, 48.0, 35.5, 27.4, 26.9, 25.7, 21.7, 18.0, -4.9, -5.0 ppm; HRMS-EI Calcd for $\mathrm{C}_{27} \mathrm{H}_{30} \mathrm{NO}_{10} \mathrm{SSi}\left(\mathrm{M}^{+}-57\right)$ : 588.1359, Found: 588.1354; Anal. calcd for $\mathrm{C}_{31} \mathrm{H}_{39} \mathrm{NO}_{10} \mathrm{SSi}$ C, 57.65; H, 6.09; found C, 58.01; H, 6.37 


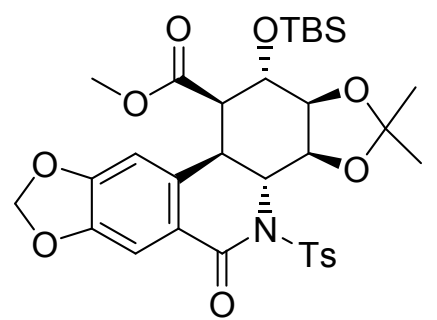

(3aS,3bR,10bR,11R,12S,12aS)-12-(tert-Butyl-dimethyl-silanyloxy)-2,2-dimethyl-5oxo-4-(toluene-4-sulfonyl)-3a,3b,4,5,10b,11,12,12a-octahydro-1,3,7,9-tetraoxa-4-azadicyclopenta $[a, h]$ phenanthrene-11-carboxylic acid methyl ester (18). To a solution of carboxylic acid $17(45 \mathrm{mg}, 0.069 \mathrm{mmol})$ in diethyl ether $(3 \mathrm{~mL})$ was added freshly prepared diazomethane solution in diethyl ether until the persistence of yellow color and total consumption of starting material (by TLC). The reaction was quenched with one drop of acetic acid followed by saturated sodium bicarbonate solution $(1 \mathrm{~mL})$, diluted with diethyl ether $(30 \mathrm{~mL})$ and washed with saturated sodium bicarbonate solution $(2 \times 1$ $\mathrm{mL}$ ), dried over magnesium sulfate, filtered and concentrated. The crude reaction mixture was passed through short silica plug using hexane/ethyl acetate $1: 1$ as eluent and concentrated to provide methyl ester $\mathbf{1 8}$ that was used without further purification. Yield: $38 \mathrm{mg}, 83 \%$, white crystalline solid; $R_{\mathrm{f}} 0.45$ (hexanes:ethyl acetate, $1: 1$ ); $\mathrm{mp}>200{ }^{\circ} \mathrm{C}$; $[\alpha]_{\mathrm{D}}^{22}-25.6809\left(c 0.75, \mathrm{CHCl}_{3}\right)$; IR $(\mathrm{KBr}) v 2986,2953,2931,2896,2858,1739,1692$, $1620,1598,1505,1485,1361,1289,1264,1173 \mathrm{~cm}^{-1} ;{ }^{1} \mathrm{H}$ NMR $\left(300 \mathrm{MHz}, \mathrm{CDCl}_{3}\right) \delta$ : $8.30(\mathrm{~d}, J=8.4 \mathrm{~Hz}, 2 \mathrm{H}), 7.55(\mathrm{~s}, 1 \mathrm{H}), 7.32(\mathrm{~d}, J=8.3 \mathrm{~Hz}, 2 \mathrm{H}), 6.58(\mathrm{~s}, 1 \mathrm{H}), 6.02(\mathrm{~s}, 2 \mathrm{H})$, $5.78(\mathrm{dd}, J=8.30 \mathrm{~Hz}, J=5.4 \mathrm{~Hz}, 1 \mathrm{H}), 4.9(\mathrm{dd}, J=12.5 \mathrm{~Hz}, J=8.3 \mathrm{~Hz}, 1 \mathrm{H}), 4.78$ (t, $J=$ $3.0 \mathrm{~Hz}, 1 \mathrm{H}), 4.24(\mathrm{dd} J=5.36 \mathrm{~Hz}, J=2.9 \mathrm{~Hz}, 1 \mathrm{H}), 3.79$ (dd, $J=12.4, J=4.2 \mathrm{~Hz}, 1 \mathrm{H}$ ), $3.56(\mathrm{~s}, 3 \mathrm{H}), 3.40(\mathrm{t}, J=3.7 \mathrm{~Hz}, 1 \mathrm{H}), 2.45(\mathrm{~s}, 3 \mathrm{H}), 1.41(\mathrm{~s}, 3 \mathrm{H}), 1.35(\mathrm{~s}, 1 \mathrm{H}), 0.98(\mathrm{~s}$, 9H), $0.24(\mathrm{~s}, 3 \mathrm{H}), 0.23(\mathrm{~s}, 3 \mathrm{H}) \mathrm{ppm} ;{ }^{13} \mathrm{C}$ NMR $\left(75 \mathrm{MHz}, \mathrm{CDCl}_{3}\right) \delta: 169.4,166.3,152.8$, $146.8,143.7,139.0,138.2,128.9,128.8,122.4,109.8,109.2$, 103.5, 102.0, 72.9, 68.2, 65.2, 51.9, 48.1, 35.9, 27.5, 26.8, 25.7, 21.6, 18.0, -4.8, -4.9 ppm; HRMS-EI Calcd for $\mathrm{C}_{28} \mathrm{H}_{32} \mathrm{NO}_{10} \mathrm{SSi}\left(\mathrm{M}^{+}-57\right): 602.1516$, Found: 602.1516 


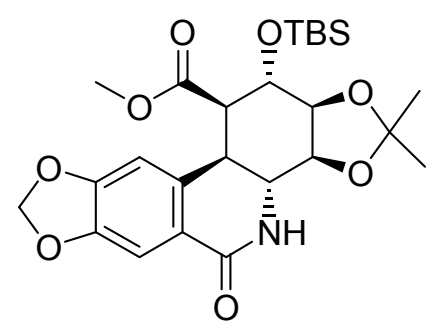

(3aS,3bR,10bR,11R,12S,12aS)-12-(tert-Butyl-dimethyl-silanyloxy)-2,2-dimethyl-5oxo-3a,3b,4,5,10b,11,12,12a-octahydro-1,3,7,9-tetraoxa-4-aza dicyclopenta $[a, h]$ phenanthrene-11-carboxylic acid methyl ester. To a solution of $\mathbf{1 8}$ $(52 \mathrm{mg}, 0.079 \mathrm{mmol})$ in dry THF $(1 \mathrm{~mL})$ at $-50{ }^{\circ} \mathrm{C}$ was added a $0.5 \mathrm{M}$ solution of $\mathrm{Na} /$ naphthalene in DME until a green color persisted and total consumption of starting material was observed (by TLC). The solution was stirred for 10 minutes before the reaction was quenched with saturated aqueous ammonium chloride solution $(1 \mathrm{~mL})$. The reaction was warmed to room temperature and extracted with $\mathrm{CH}_{2} \mathrm{Cl}_{2}(6 \times 15 \mathrm{~mL})$. The combined organic phase was dried over sodium sulfate, filtered, and concentrated. The final product was isolated by column chromatography (hexanes:ethyl acetate, 5:1 to 2:1). Yield: $23 \mathrm{mg}, 58 \%$, clear oil; $R_{\mathrm{f}} 0.28$ (hexanes:ethyl acetate, $\left.1: 1\right) ;[\alpha]_{\mathrm{D}}{ }^{22}-14.51$ (c 0.50, $\mathrm{CHCl}_{3}$ ); IR (film) v3320, 2952, 2930, 2895, 2857, 1743, 1669, 1619, 1504, 1484, $14601385,1369,1321,1288,1260,1222 \mathrm{~cm}^{-1} ;{ }^{1} \mathrm{H}$ NMR $\left(600 \mathrm{MHz}, \mathrm{CDCl}_{3}\right) \delta: 7.62(\mathrm{~s}$, $1 \mathrm{H}), 6.56(\mathrm{~s}, 1 \mathrm{H}), 6.02(\mathrm{~s}, 2 \mathrm{H}), 5.96(\mathrm{~s}, 1 \mathrm{H}), 4.86(\mathrm{t}, J=2.6,1 \mathrm{H}), 4.41(\mathrm{dd}, J=13.6 \mathrm{~Hz}$, $J=8.2 \mathrm{~Hz}, 1 \mathrm{H}), 4.18(\mathrm{dd}, J=8.25 \mathrm{~Hz}, J=4.8 \mathrm{~Hz}, 1 \mathrm{H}), 4.11(\mathrm{~m}, 1 \mathrm{H}), 3.66(\mathrm{~s}, 3 \mathrm{H}), 3.40$ (dd, $J=13.6 \mathrm{~Hz}, J=3.7 \mathrm{~Hz}, 1 \mathrm{H}), 3.33(\mathrm{~m}, 1 \mathrm{H}), 2.06(\mathrm{~s}, 1 \mathrm{H}), 1.40(\mathrm{~s}, 3 \mathrm{H}), 1.37(\mathrm{~s}, 3 \mathrm{H})$, $0.92(\mathrm{~s}, 9 \mathrm{H}), 0.21(\mathrm{~s}, 3 \mathrm{H}), 0.20(\mathrm{~s}, 3 \mathrm{H}) \mathrm{ppm} ;{ }^{13} \mathrm{C} \mathrm{NMR}(150 \mathrm{MHz}, \mathrm{CDCl} 3) \delta: 169.6$, 165.4, 151.4, 146.6, 135.4, 122.6, 110.5, 108.6, 103.3, 101.7, 69.2, 53.1, 51.9, 45.9, 33.4, 27.6, 26.5, 25.7, 17.9, -4.9, -5.0 ppm; HRMS-EI Calcd for $\mathrm{C}_{25} \mathrm{H}_{35} \mathrm{NO}_{8} \mathrm{Si}\left(\mathrm{M}^{+}\right)$: 505.2132, Found: 505.2131 
<smiles>COC(=O)[C@@H]1[C@H](O)[C@@H](O)[C@@H](O)[C@@H]2NC(=O)c3cc4c(cc3[C@@H]12)OCO4</smiles>

(1R,2S,3R,4S,4aR,11bR)-2,3,4-Trihydroxy-6-oxo-1,2,3,4,4a,5,6,11b-octahydro[1,3]dioxolo[4,5-j]phenanthridine-1-carboxylic acid methyl ester (19). To a solution of the detosylated methyl ester $(23 \mathrm{mg}, 0.046 \mathrm{mmol})$ in methanol $(2 \mathrm{~mL})$ was added $3 \%$ $\mathrm{HCl}$ in methanol $(0.5 \mathrm{~mL})$. The reaction mixture was stirred until total consumption of starting material (3 days). The solvent was removed under reduced pressure and the residue was purified by flash column chromatography using a 30:1 to 20:1 gradient of methylene chloride:methanol as eluent to provide methyl ester $19(11 \mathrm{mg}, 69 \%)$ as a white crystalline solid.; $\mathrm{mp}>200{ }^{\circ} \mathrm{C}$ (methylene chloride: methanol); $R_{\mathrm{f}} 0.06$ (methylene chloride/methanol, 20:1); $[\alpha]_{\mathrm{D}}^{22}+24.53$ (c 0.25, MeOH); IR (KBr) v3311, 2913, 1732,

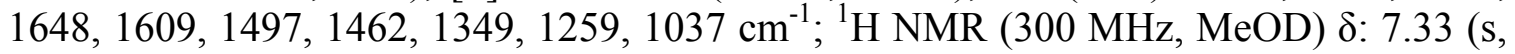
$1 \mathrm{H}), 6.59(\mathrm{~s}, 1 \mathrm{H}), 5.93(\mathrm{~d}, J=3.7,2 \mathrm{H}), 4.50(\mathrm{t}, J=3.12,1 \mathrm{H}), 4.21(\mathrm{dd}, J=13.1 \mathrm{~Hz}, J=$ $10.1 \mathrm{~Hz}, 1 \mathrm{H}), 3.86(\mathrm{~m}, 1 \mathrm{H}), 3.79,(\mathrm{dd}, J=10.1, J=3.0,1 \mathrm{H}), 3.51(\mathrm{~s}, 3 \mathrm{H}), 3.39(\mathrm{~m}, 1 \mathrm{H})$, $3.29(\mathrm{dd}, J=13.1, J=4.1,1 \mathrm{H}) \mathrm{ppm} ;{ }^{13} \mathrm{C} \mathrm{NMR}(75 \mathrm{MHz}, \mathrm{MeOD}) \delta: 170.8,166.4,151.7$, 146.4, 137.3, 121.7, 106.9, 103.7, 101.8, 72.2, 71.9, 70.9, 51.4, 50.6, 44.8, 35.4 ppm; HRMS-FAB: $(\mathrm{m} / z)(\mathrm{M}+\mathrm{H})^{+}$: Calcd for $\mathrm{C}_{16} \mathrm{H}_{17} \mathrm{NO}_{8}: 352.1032$, Found: 352.0941<smiles>O=C1N[C@H]2[C@H](O)[C@H](O)[C@@H](O)[C@H](C(=O)O)[C@H]2c2cc3c(cc21)OCO3</smiles>

(1R,2S,3R,4S,4aR,11bR)-2,3,4-Trihydroxy-6-oxo-1,2,3,4,4a,5,6,11b-octahydro[1,3]dioxolo[4,5-j]phenanthridine-1-carboxylic acid (20). To a solution of 19 (6 mg, $0.017 \mathrm{mmol})$ in methanol $(0.5 \mathrm{~mL})$ was added $\mathrm{LiOH}(1 \mathrm{mg}, 1.5 \mathrm{mmol})$. The reaction mixture was heated at $45{ }^{\circ} \mathrm{C}$ and stirred until total consumption of starting material (2 days) as monitored by TLC. The reaction was made slightly acidic with the addition of $\mathrm{HCl}$ (5 drops, 1M) and concentrated to provide acid $20(5 \mathrm{mg}, 95 \%)$ as a white crystalline solid.; $\mathrm{mp}>200{ }^{\circ} \mathrm{C} ; R_{\mathrm{f}} 0.06$ (methylene chloride:methanol, 4:1); IR (KBr) $v$ $3412,2920,2115,1641,1505,1471,1409,1462,1363,1267 \mathrm{~cm}^{-1} ;{ }^{1} \mathrm{H}$ NMR $(300 \mathrm{MHz}$, MeOD) $\delta: 7.41(\mathrm{~s}, 1 \mathrm{H}), 6.72(\mathrm{~s}, 1 \mathrm{H}), 6.02(\mathrm{~d}, J=3.7,2 \mathrm{H}), 4.64(\mathrm{t}, J=3.12,1 \mathrm{H}), 4.35$ $(\mathrm{dd}, J=13.1 \mathrm{~Hz}, J=10.1 \mathrm{~Hz}, 1 \mathrm{H}), 3.99(\mathrm{~m}, 1 \mathrm{H}), 3.89$, (dd, $J=10.1, J=3.0,1 \mathrm{H}), 3.45$ $(\mathrm{m}, 1 \mathrm{H}), 3.38(\mathrm{~m}, 1 \mathrm{H}) \mathrm{ppm} ;{ }^{13} \mathrm{C} \mathrm{NMR}(75 \mathrm{MHz}, \mathrm{MeOD}) \delta: 172.1,166.4,151.7,146.4$, 137.6, 121.7, 106.8, 103.8, 101.8, 72.4, 71.9, 71.1, 51.34, 45.03, 35.4 ppm; 


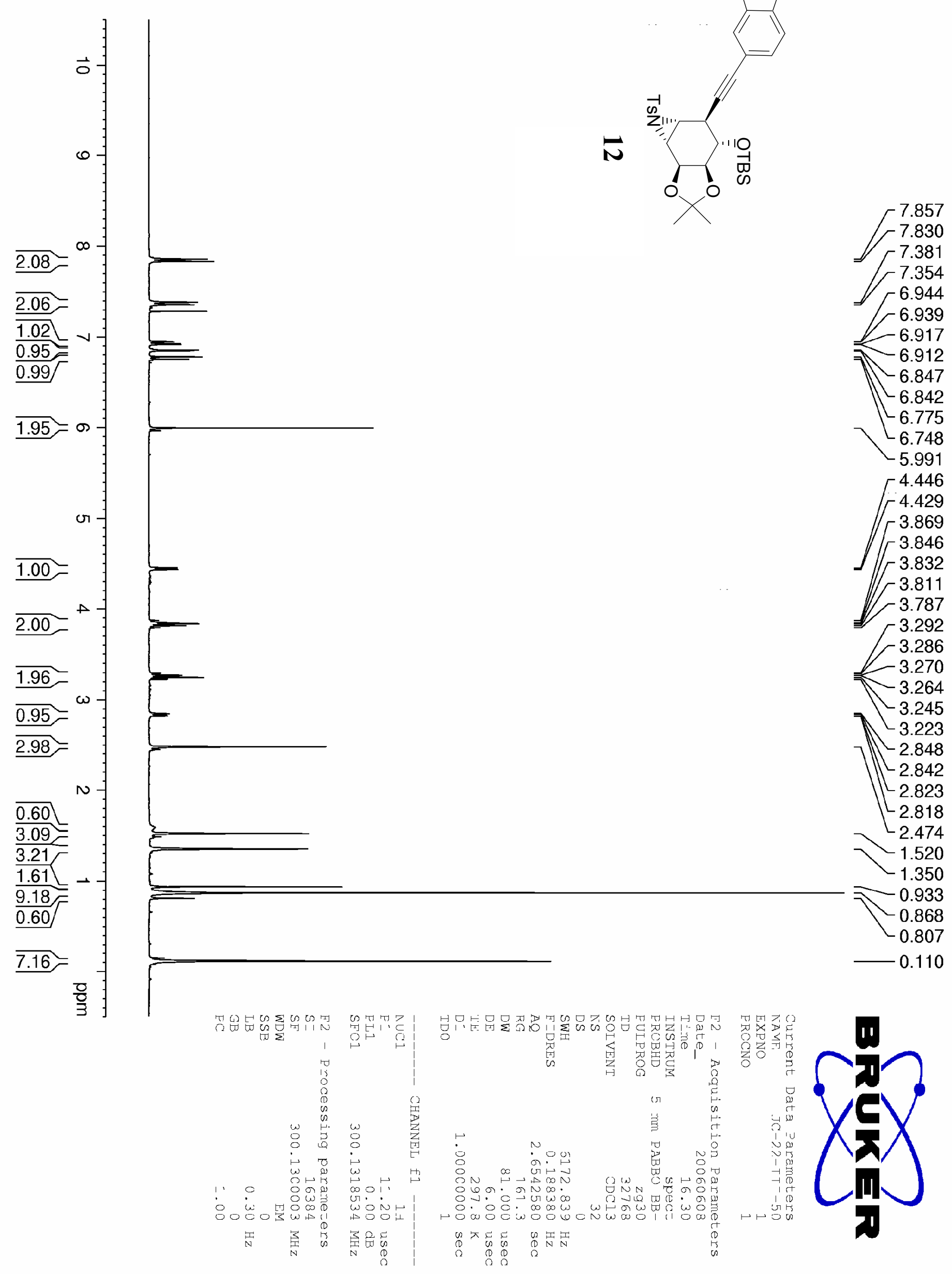




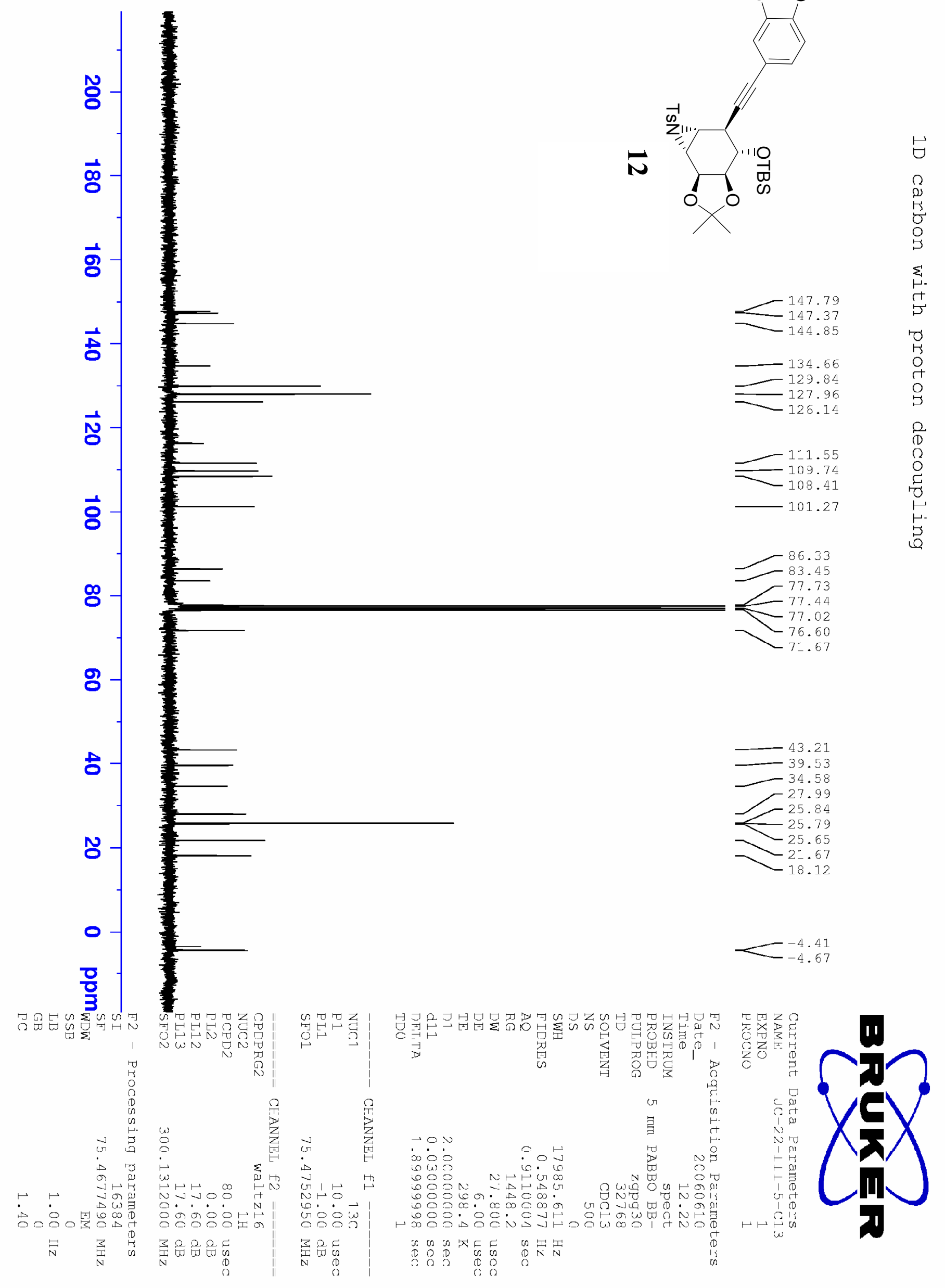




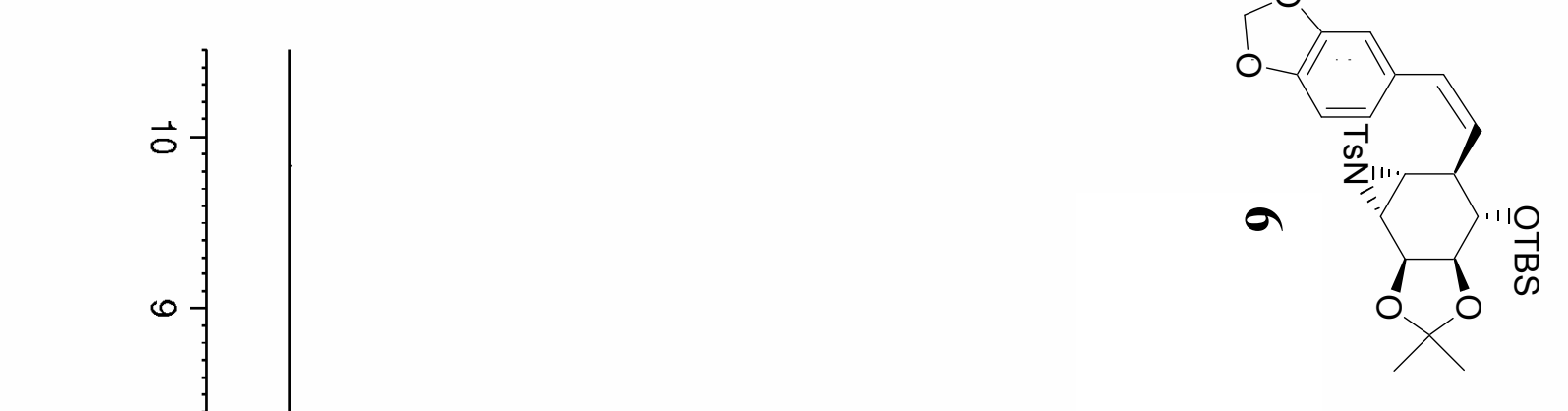

7.796
7.769
7.303
7.280
7.276
-7.706

$-6.680$

$-6.654$

3.03

$\underline{1.03}-$

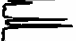

$\overline{2.17} \approx \sigma$

1.00

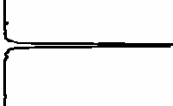

$-6.531$

$-6.492$

$-5.970$

$-5.963$

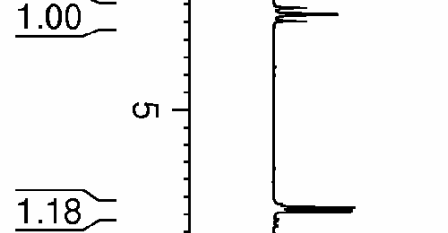

$\approx-5.577$

$-5.540$

5.502

$\widetilde{1.21}$

1.11

1.14

$\underline{\underline{2.01}} \omega$

$\underline{3.21}$

$\frac{3.87}{4.99}$

$N$

$\xi$

$\frac{\underline{9.46}}{\frac{4.45}{\frac{69}{3}}}$
$\frac{0}{3}$

$=$

$\xi$

0.785

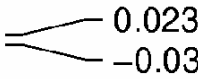

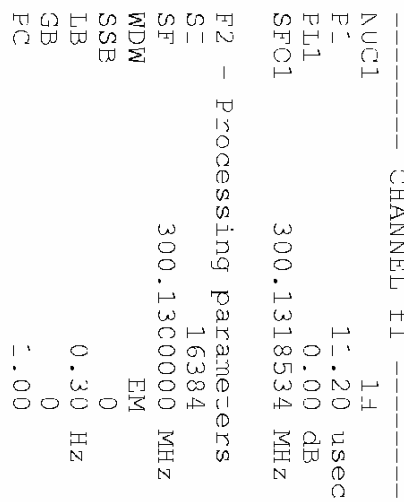

㝵

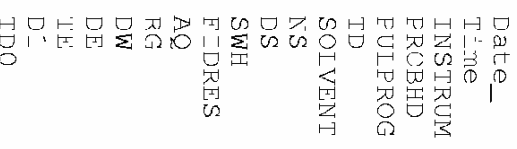

$\dot{8} \quad$ n.

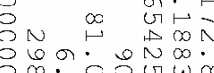

$80.00 \mathrm{~N} \omega$

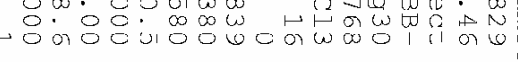

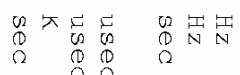

4.415

$-3.875$

$-3.853$

$-3.832$

$-3.637$

$-3.613$

$-3.589$

$-3.195$

$\approx-3.173$

$-2.960$

$\sqrt{-2.932}$

$-2.912$

2.905
2.439

1.332

$-0.03$ 


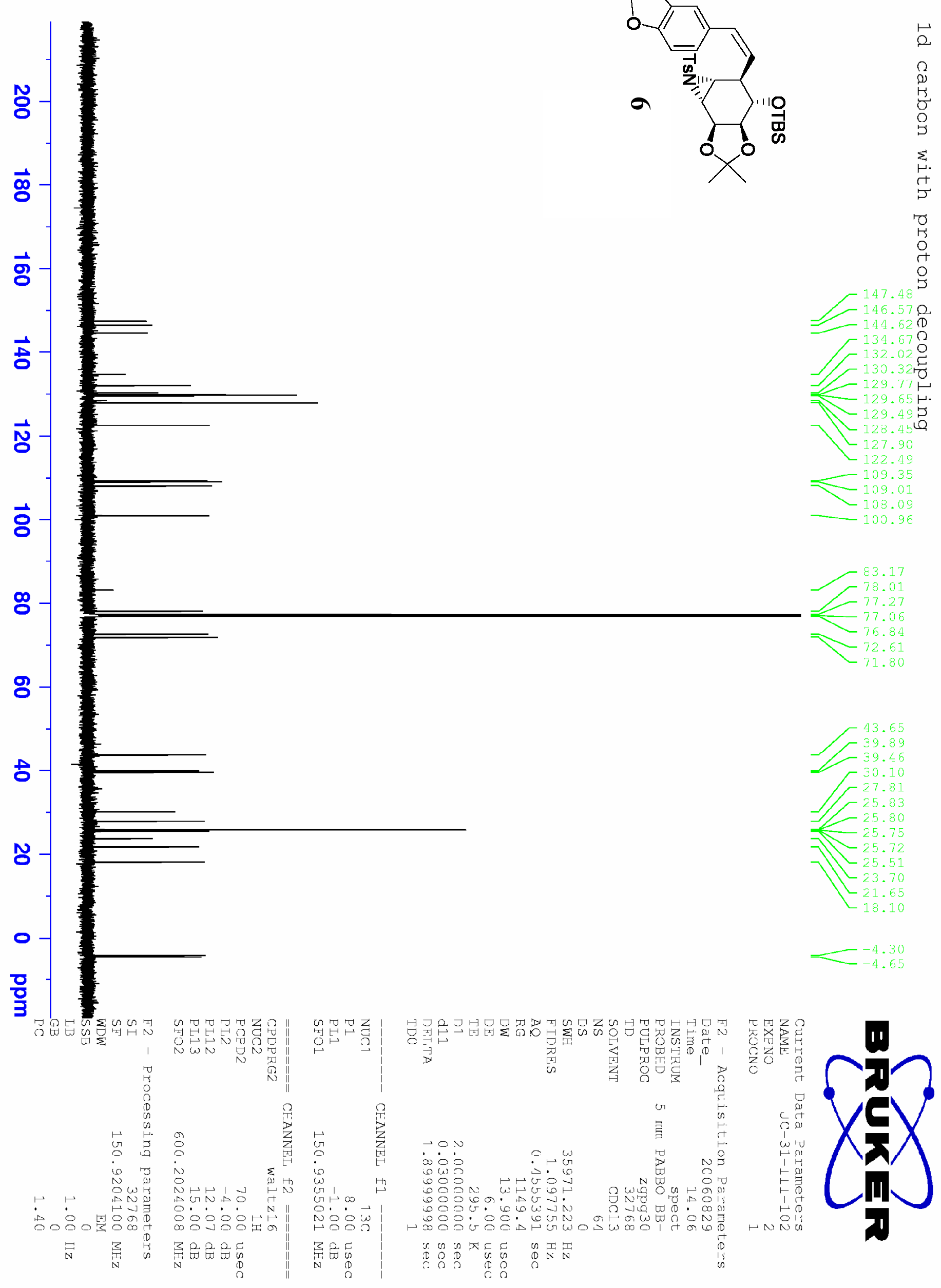




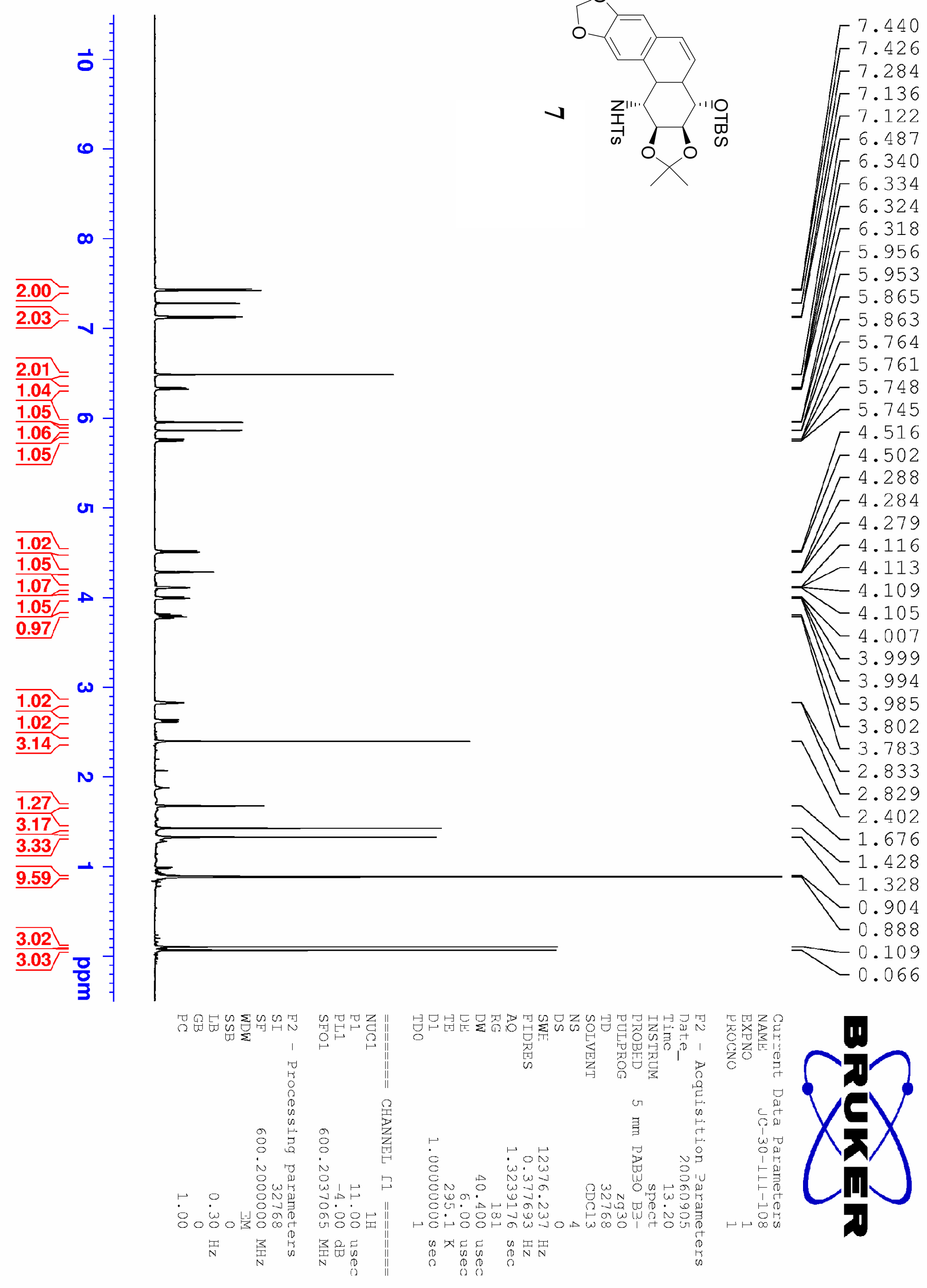




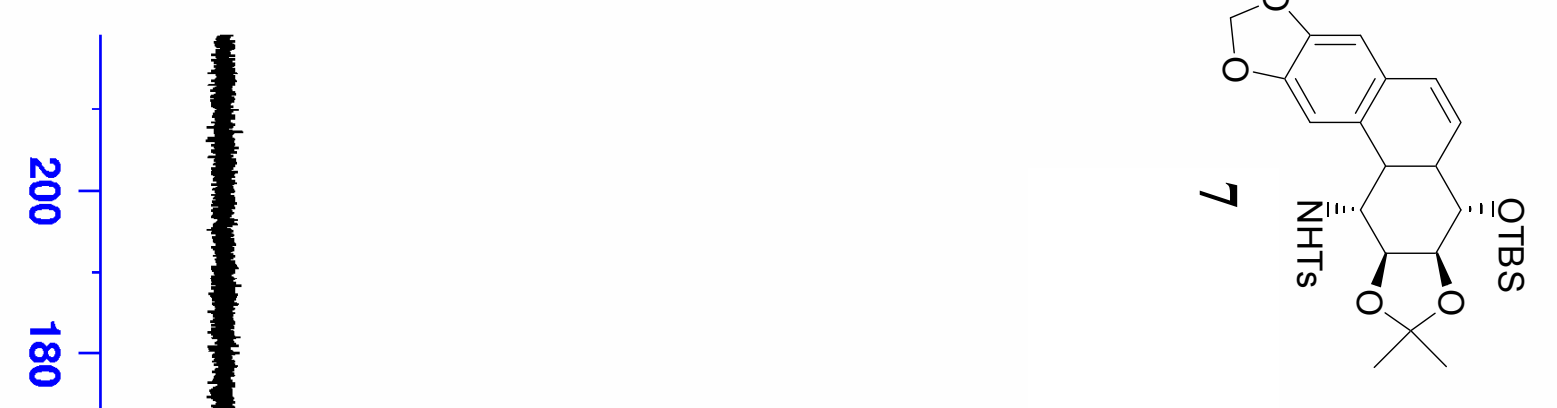

$\stackrel{\bullet}{\circ}$
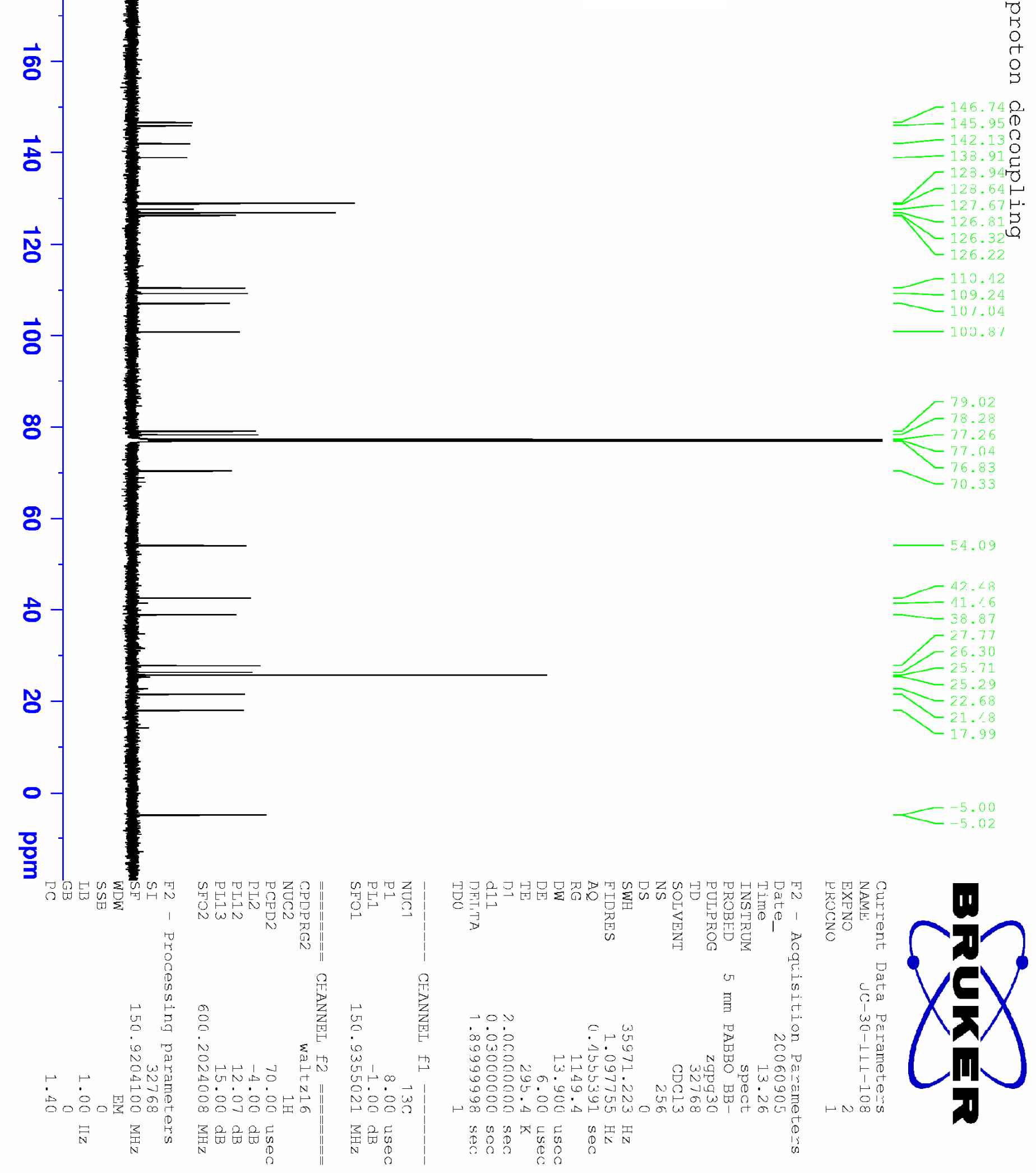


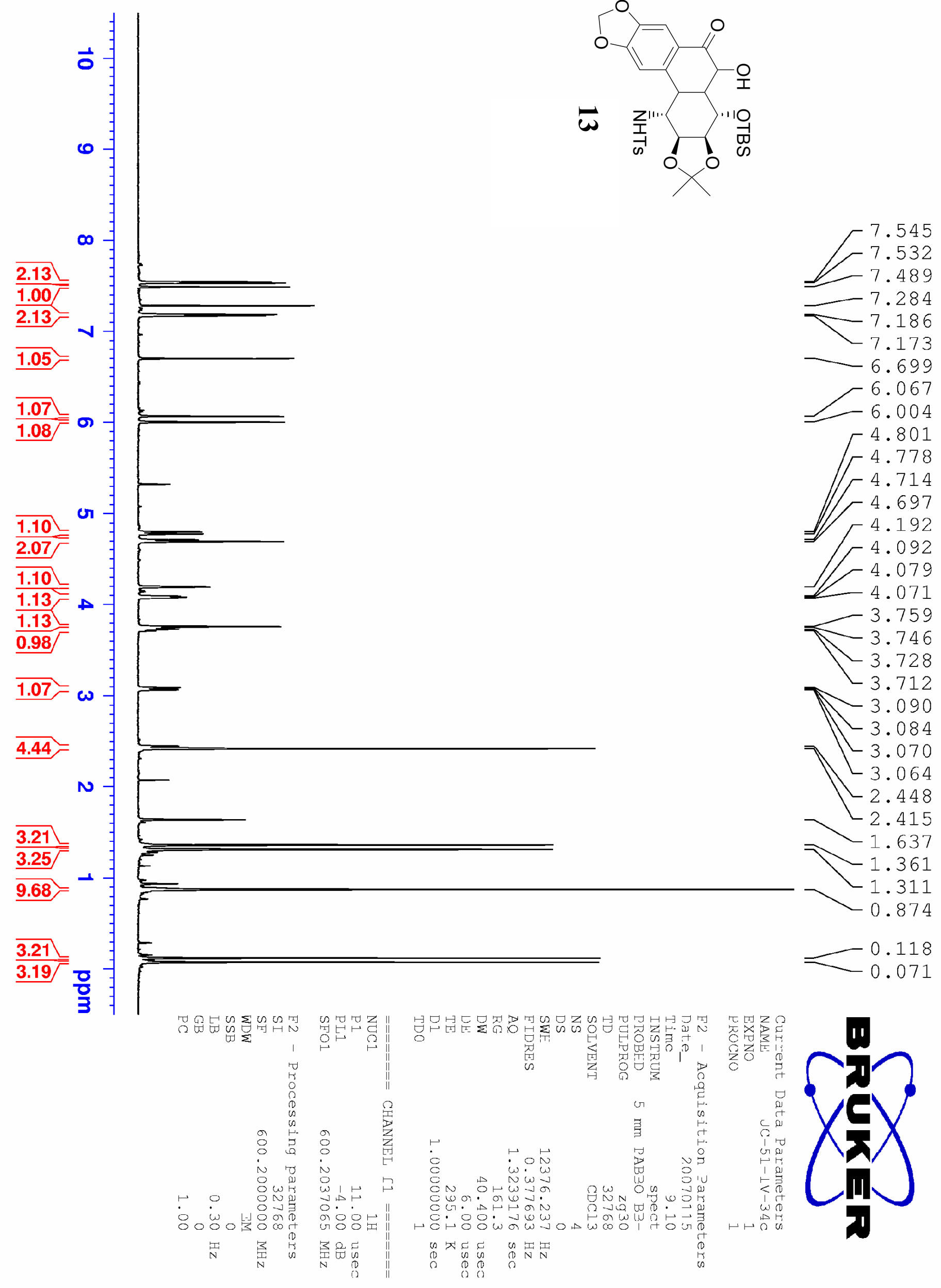



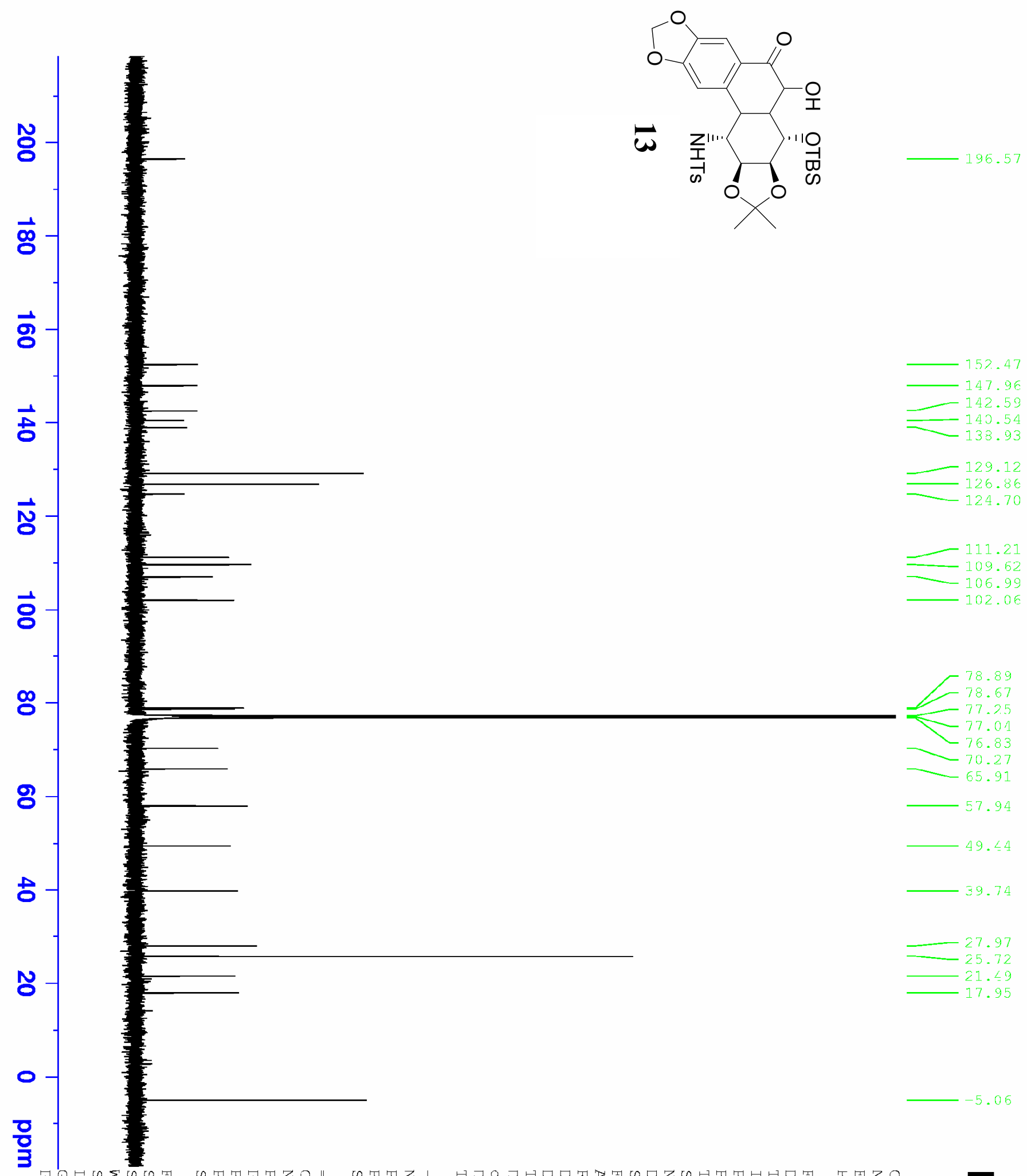

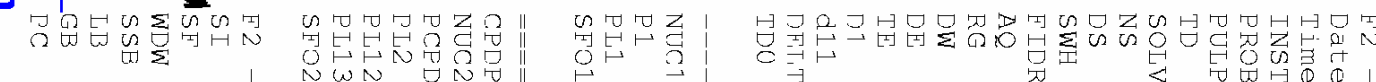

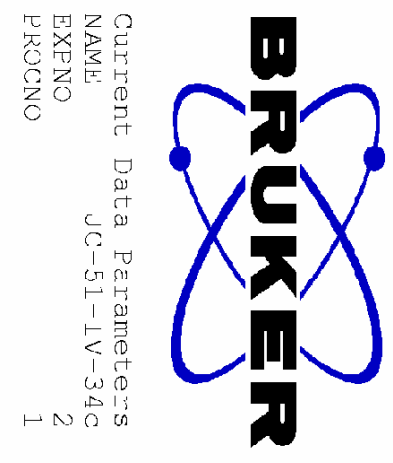

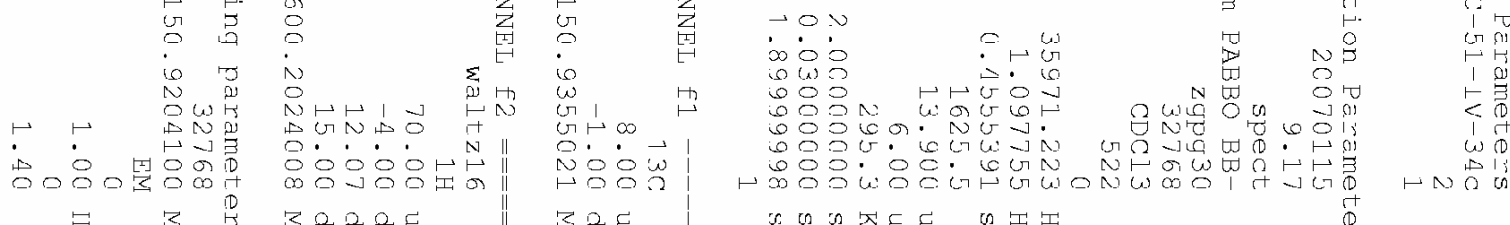

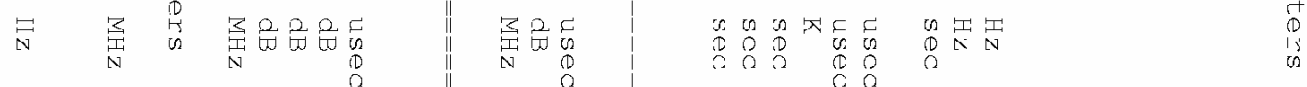




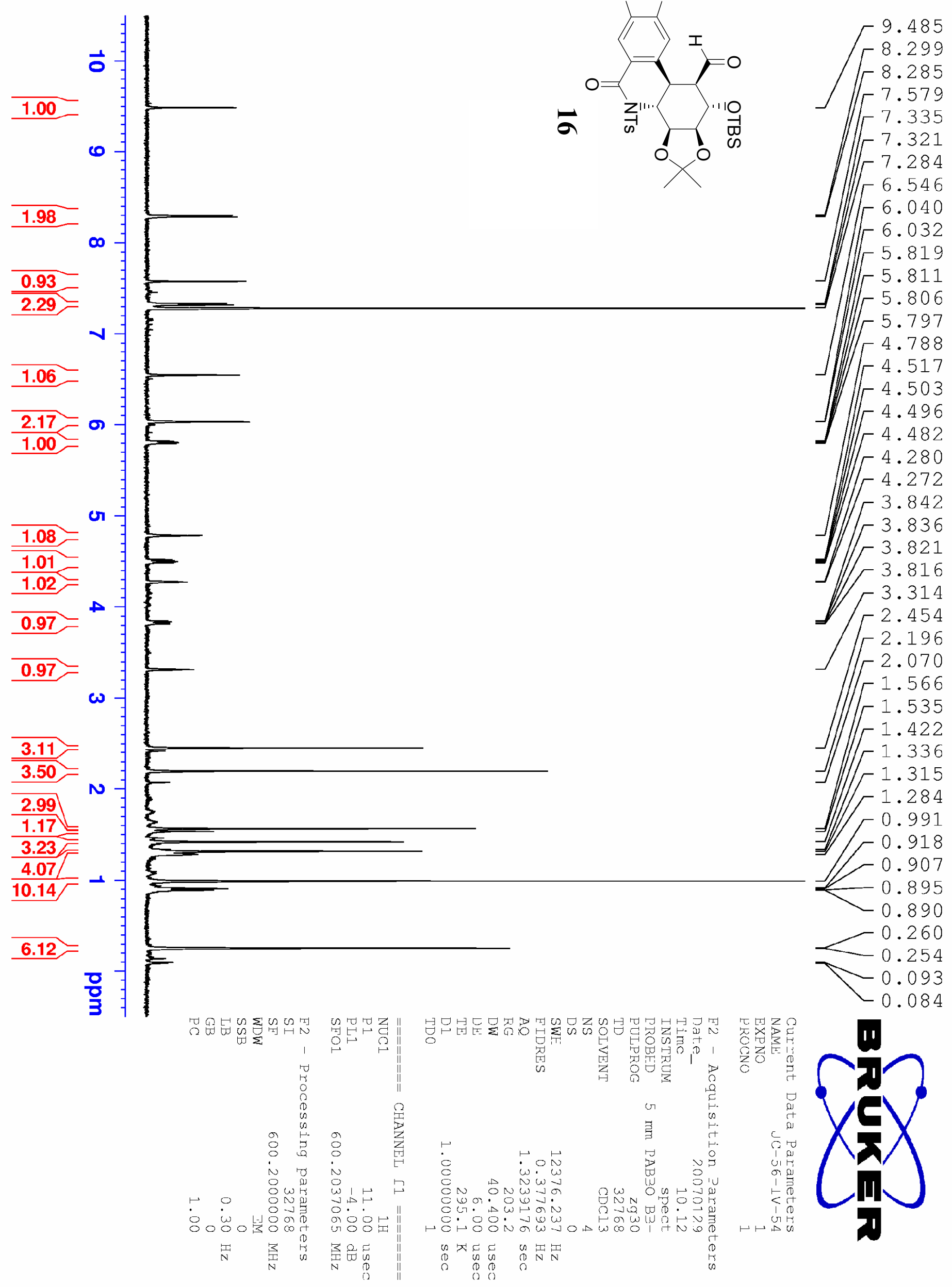



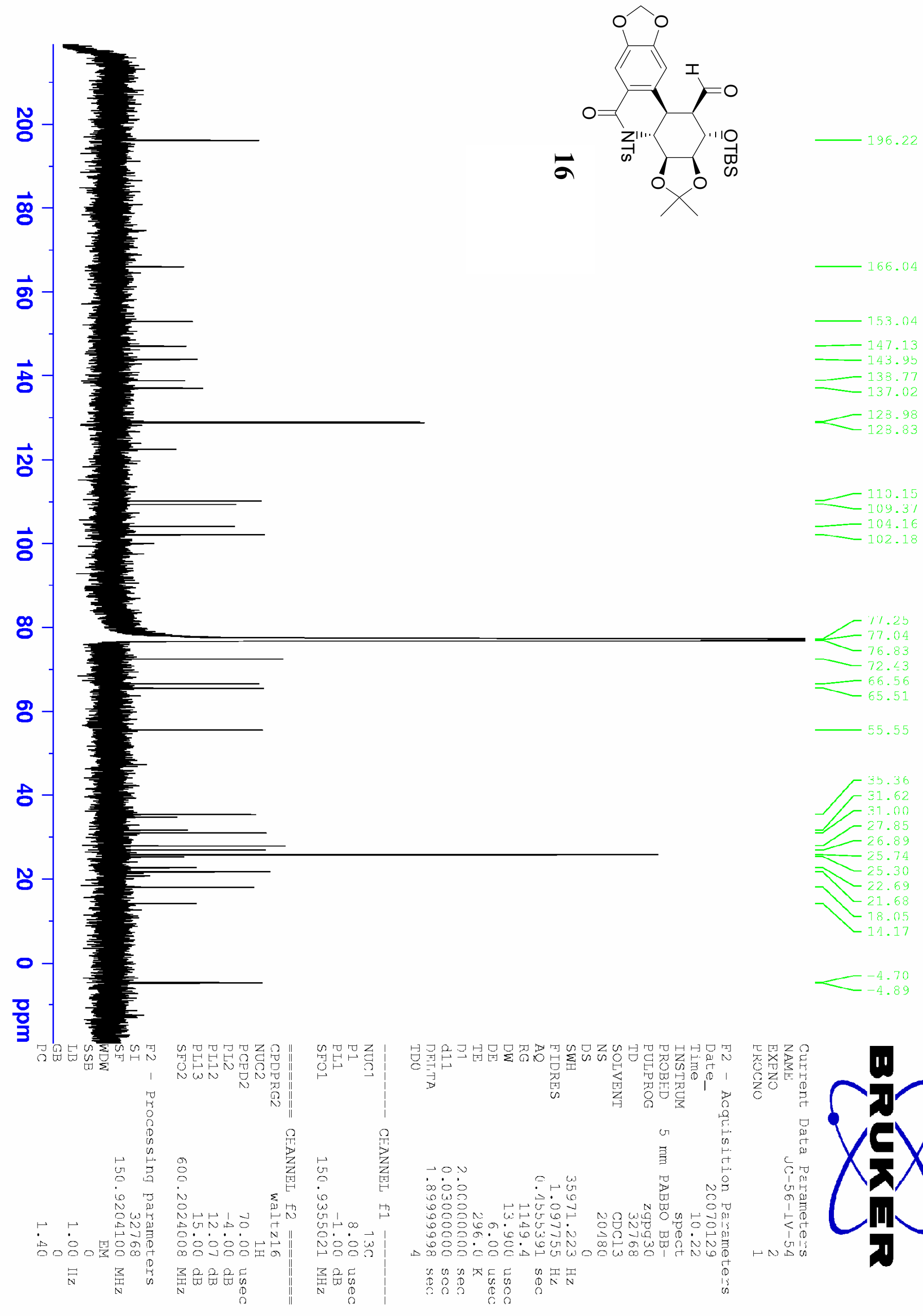


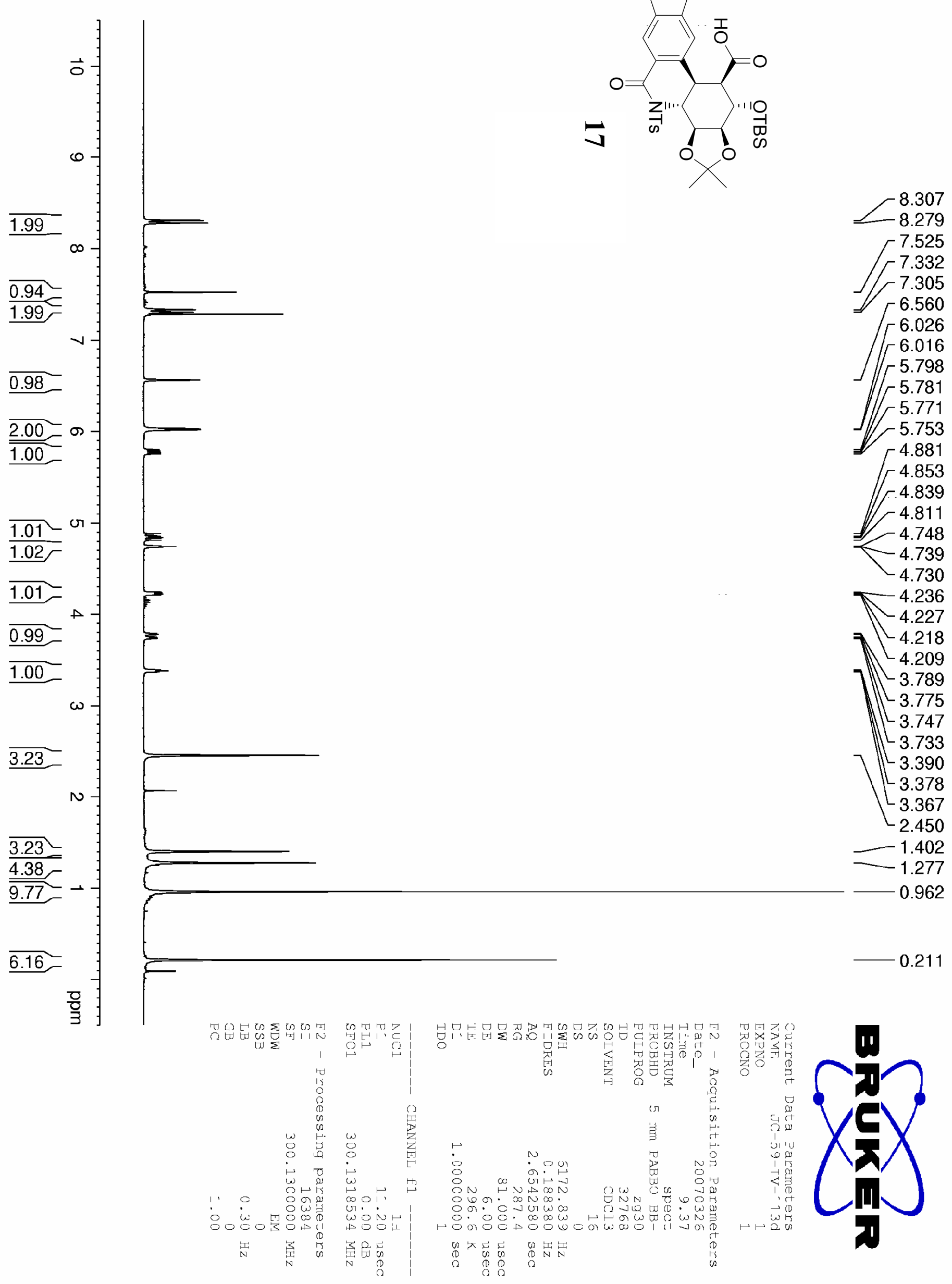



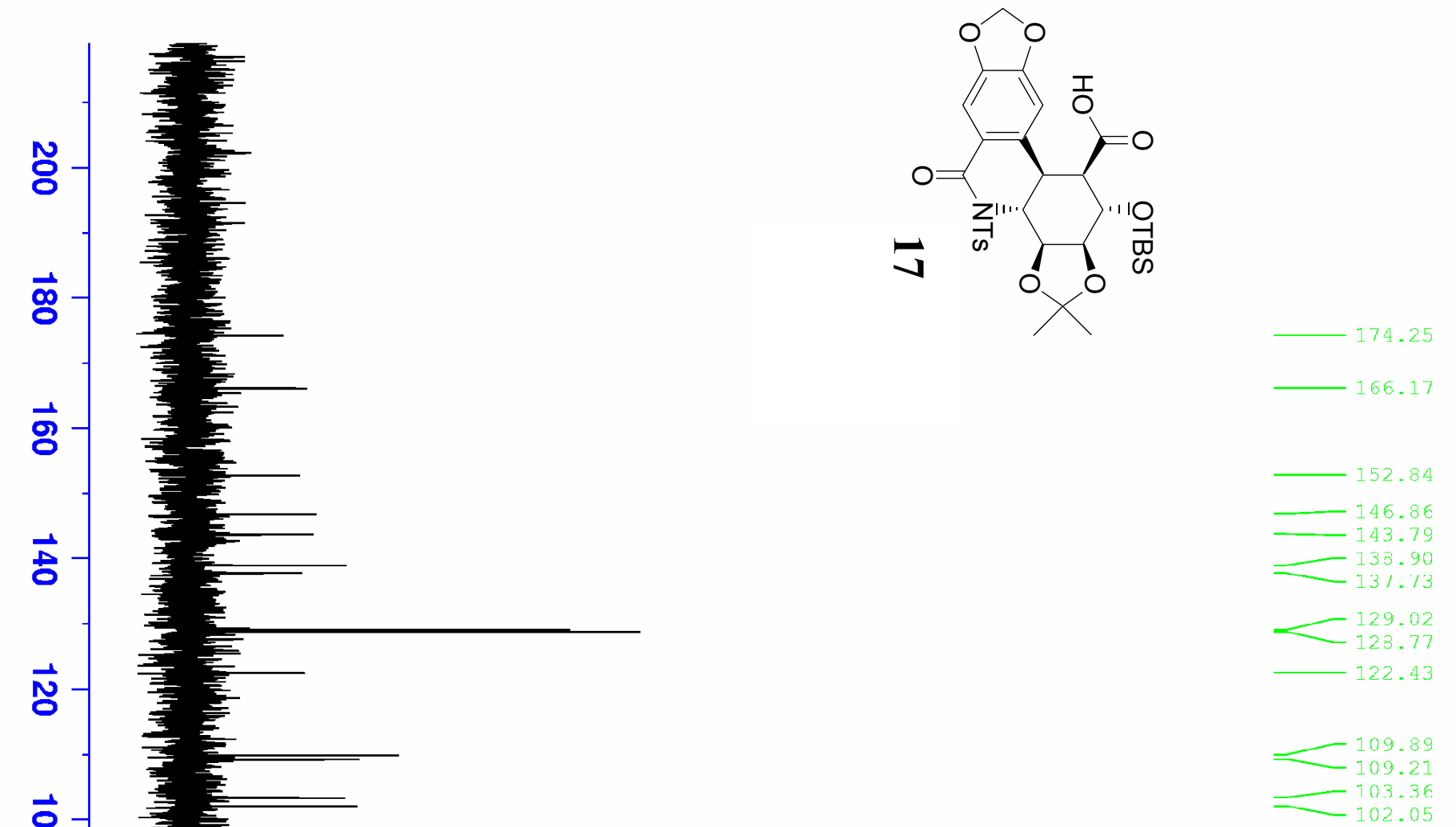

$\overrightarrow{\mathrm{s}}$

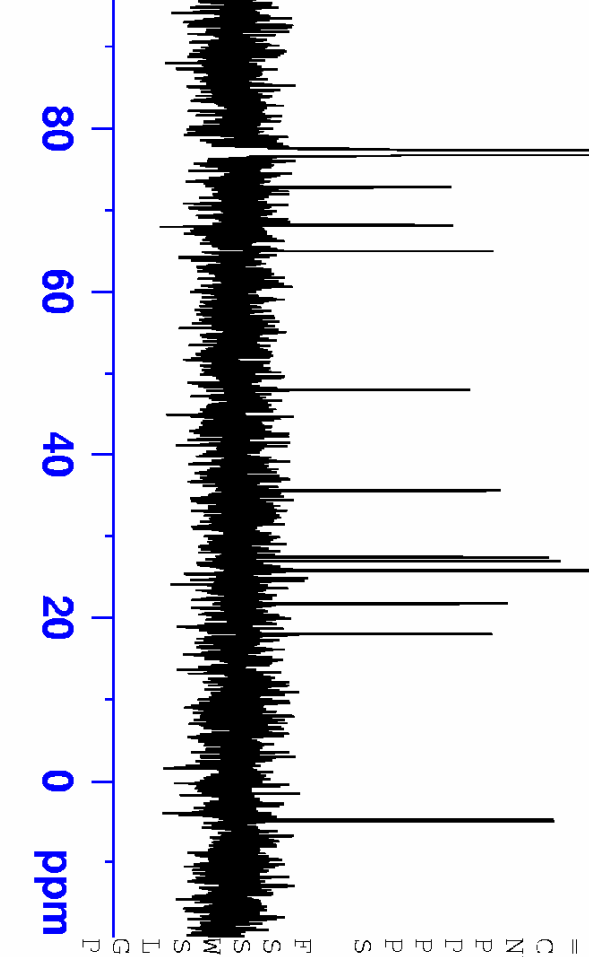

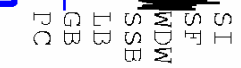

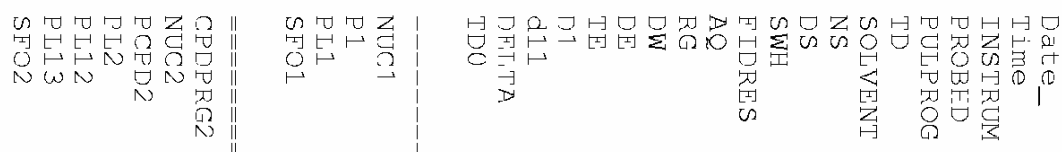

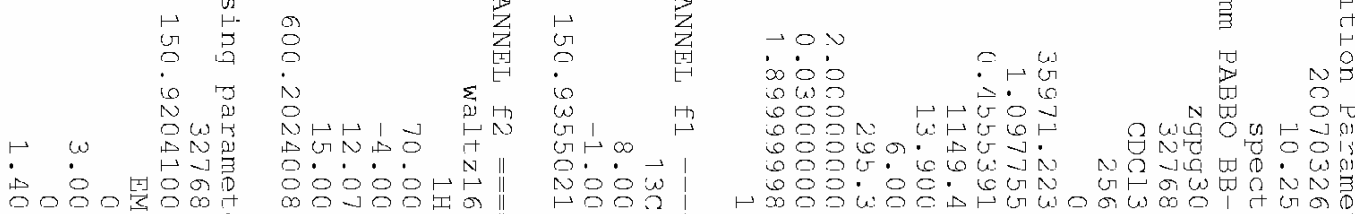
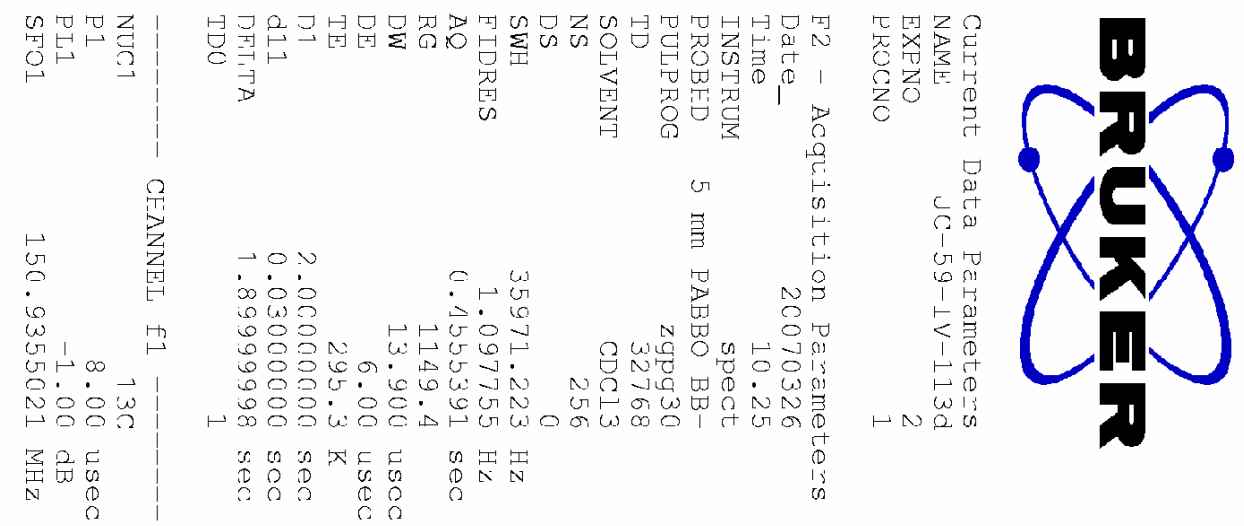
줄

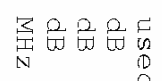




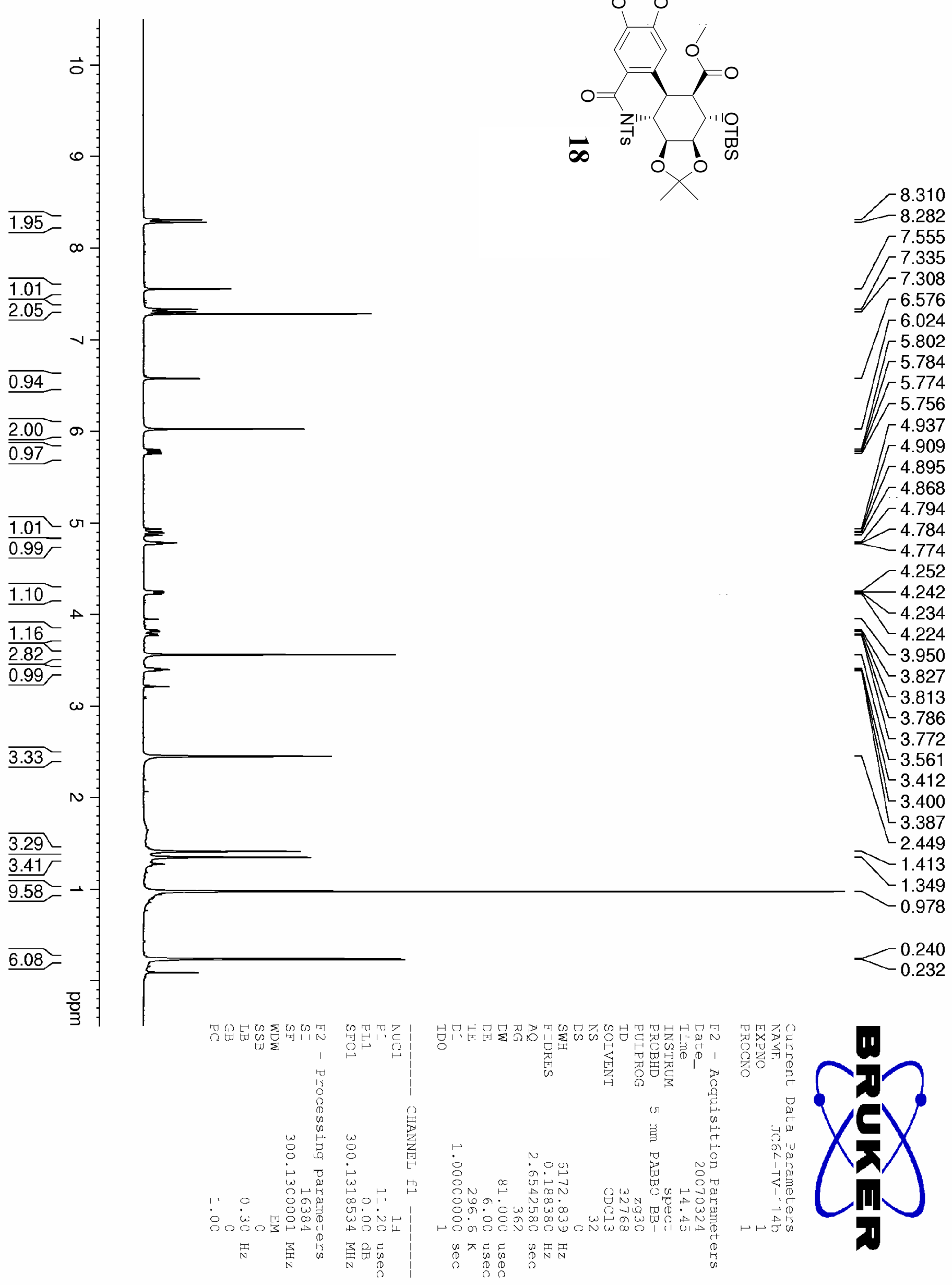



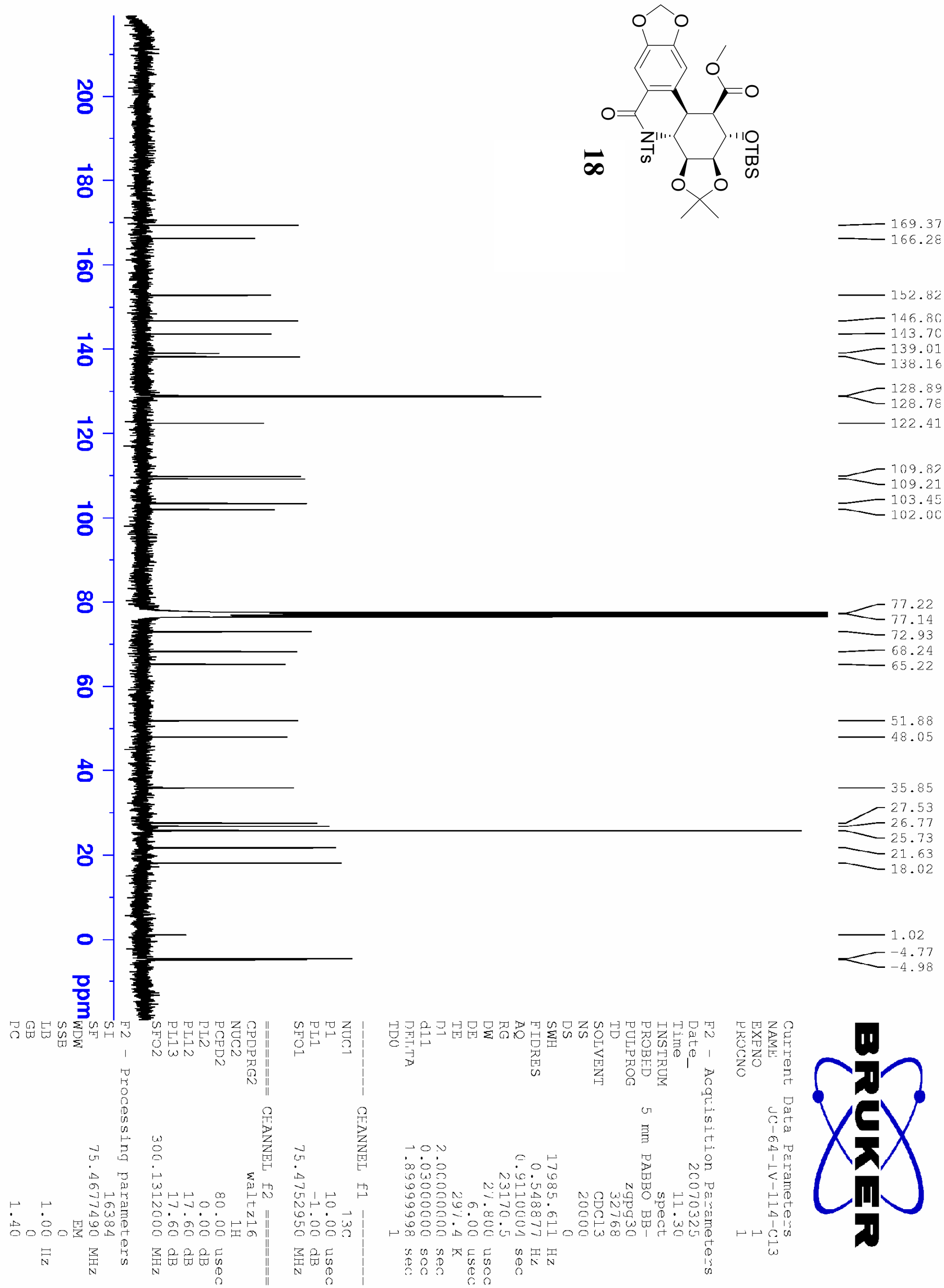

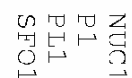

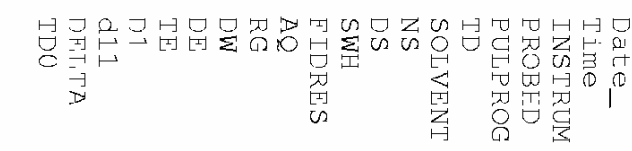
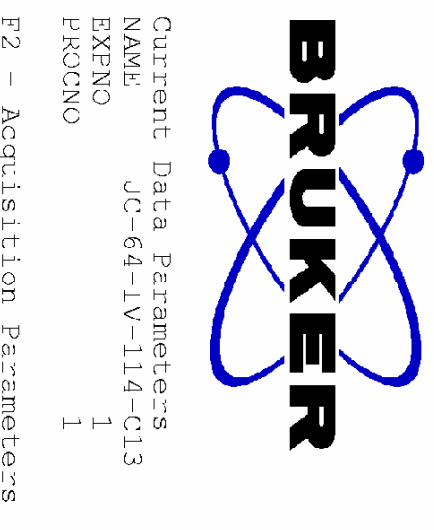


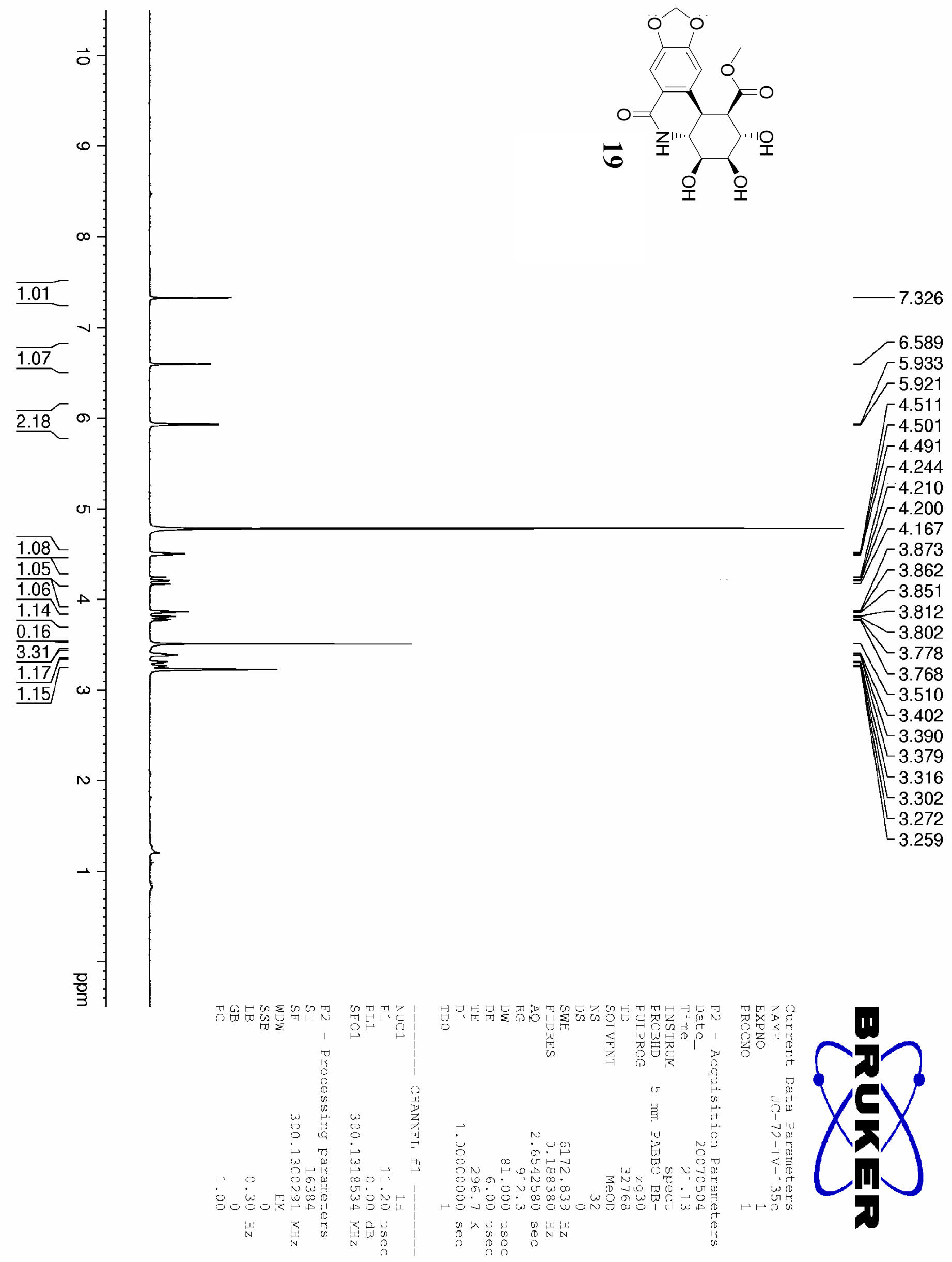




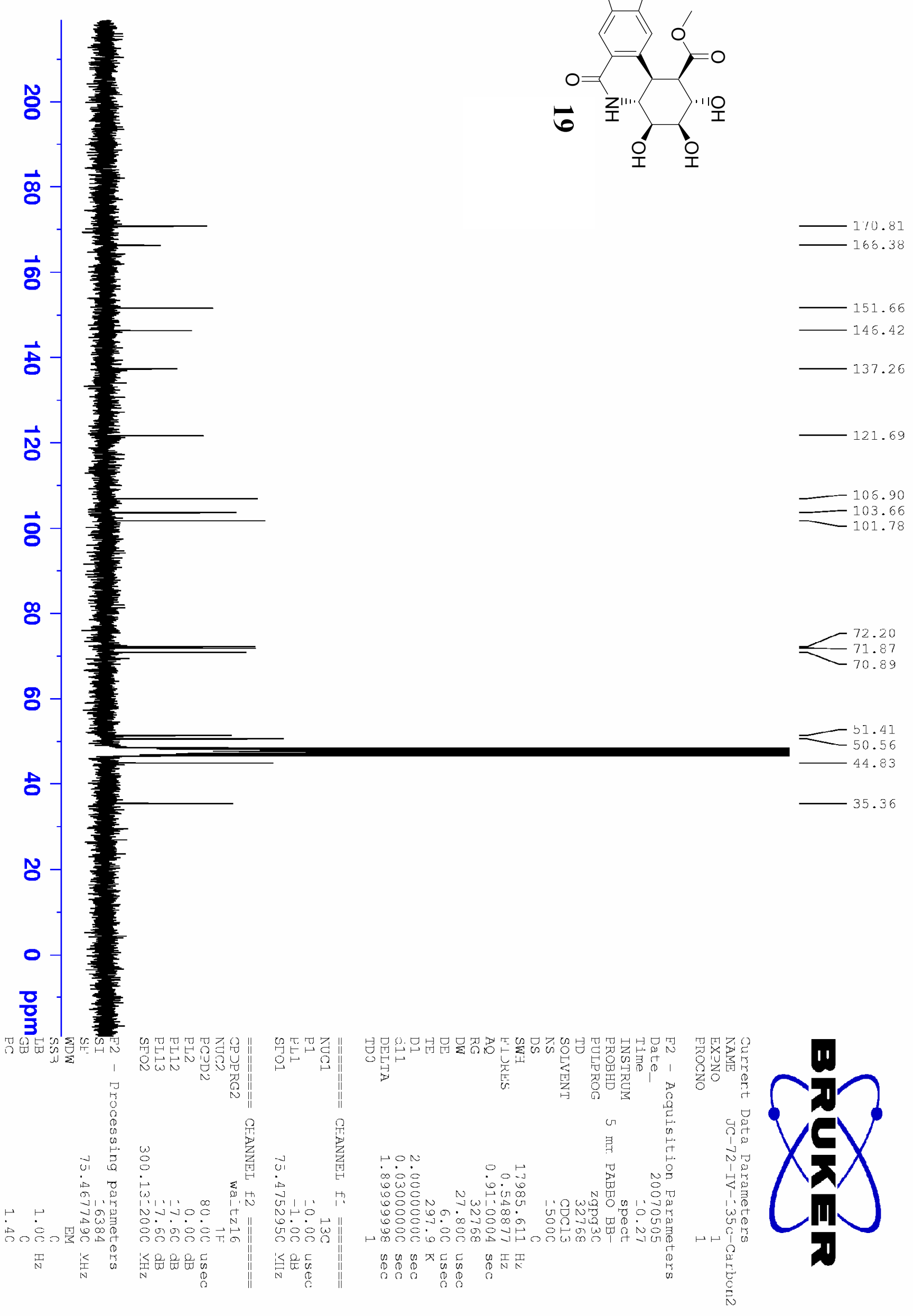




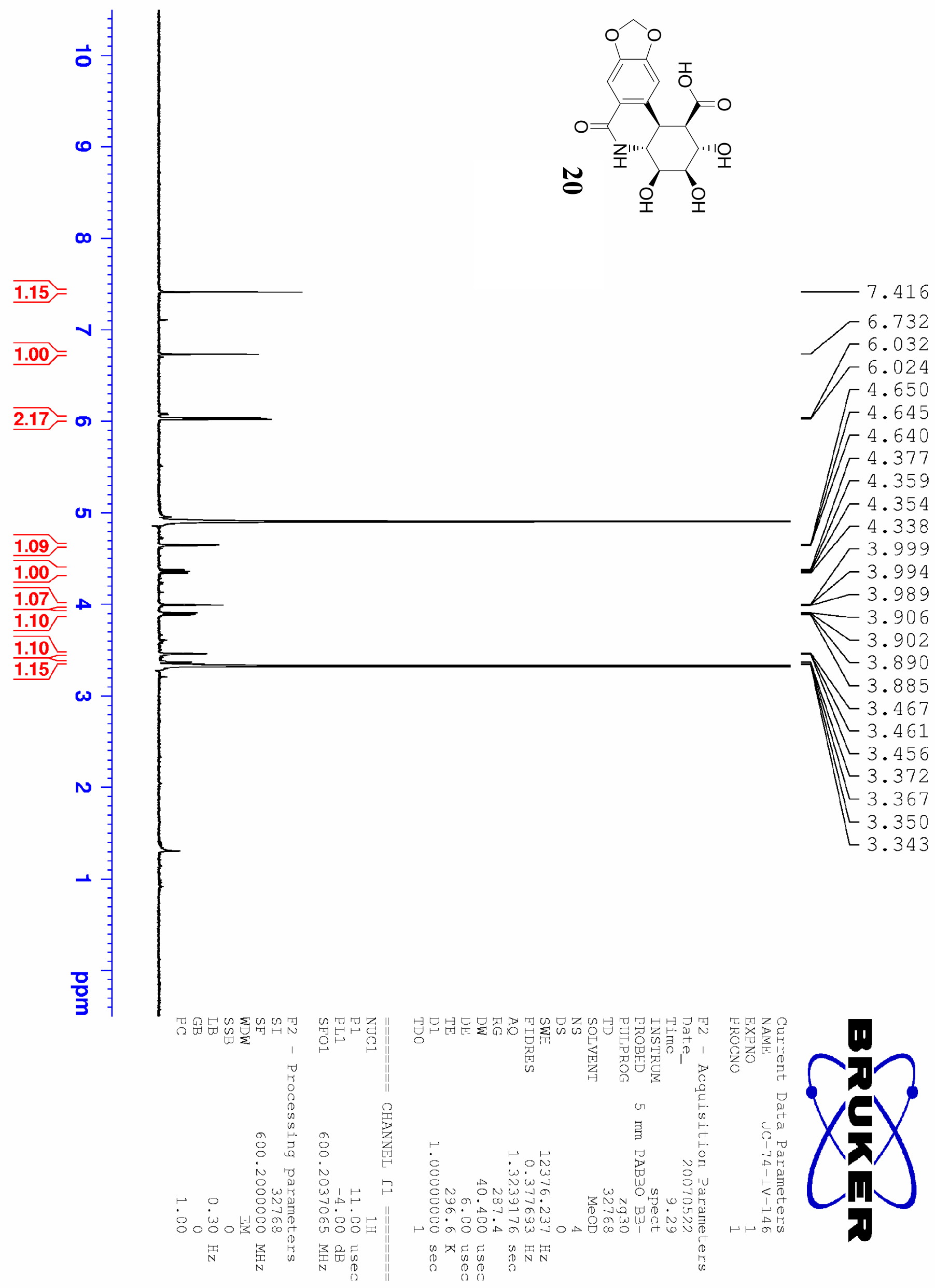




$$
\mid
$$

